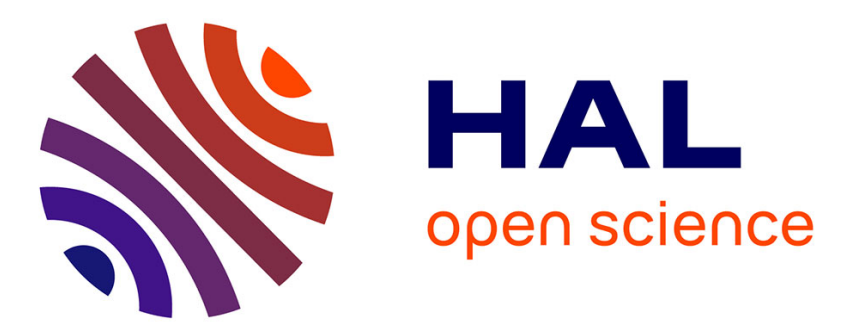

\title{
Participations ordinaires et extraordinaires. Des appropriations différenciées d'une offre institutionnelle de participation municipale \\ Guillaume Petit
}

\section{- To cite this version:}

Guillaume Petit. Participations ordinaires et extraordinaires. Des appropriations différenciées d'une offre institutionnelle de participation municipale. Participations - Revue de sciences sociales sur la démocratie et la citoyenneté, 2014, 10 (3), pp.85. 10.3917/parti.010.0085 . halshs-02948731

\section{HAL Id: halshs-02948731 \\ https://shs.hal.science/halshs-02948731}

Submitted on 3 Apr 2021

HAL is a multi-disciplinary open access archive for the deposit and dissemination of scientific research documents, whether they are published or not. The documents may come from teaching and research institutions in France or abroad, or from public or private research centers.
L'archive ouverte pluridisciplinaire HAL, est destinée au dépôt et à la diffusion de documents scientifiques de niveau recherche, publiés ou non, émanant des établissements d'enseignement et de recherche français ou étrangers, des laboratoires publics ou privés. 


\section{PARTICIPATIONS ORDINAIRES ET EXTRAORDINAIRES. DES APPROPRIATIONS DIFFÉRENCIÉES D'UNE OFFRE INSTITUTIONNELLE DE PARTICIPATION MUNICIPALE}

\section{Guillaume Petit}

De Boeck Supérieur | «Participations »

$2014 / 3 \mathrm{~N}^{\circ} 10$ | pages 85 à 120

ISSN 2034-7650

ISBN 9782804192242

Article disponible en ligne à l'adresse :

https://www.cairn.info/revue-participations-2014-3-page-85.htm

Distribution électronique Cairn.info pour De Boeck Supérieur.

(C) De Boeck Supérieur. Tous droits réservés pour tous pays.

La reproduction ou représentation de cet article, notamment par photocopie, n'est autorisée que dans les limites des conditions générales d'utilisation du site ou, le cas échéant, des conditions générales de la licence souscrite par votre établissement. Toute autre reproduction ou représentation, en tout ou partie, sous quelque forme et de quelque manière que ce soit, est interdite sauf accord préalable et écrit de l'éditeur, en dehors des cas prévus par la législation en vigueur en France. Il est précisé que son stockage dans une base de données est également interdit. 


\title{
Participations ordinaires et extraordinaires. Des appropriations différenciées d'une offre institutionnelle de participation municipale
}

\author{
> Guillaume Petit
}

\section{> Résumé}

Quelles sont les implications et les significations d'une offre de participation municipale ? À travers l'étude des appropriations différenciées d'une offre institutionnelle par les participants, nous interrogeons à quelles conditions, et dans quelle mesure, une telle offre peut convaincre des citoyens d'y prendre part. L'analyse des caractéristiques et des trajectoires des publics des dispositifs permet de contextualiser les innovations participatives dans un ordinaire de l'engagement local, et d'esquisser les conditions d'une participation non uniquement préfigurée dans les systèmes d'interconnaissance et de reconnaissance préalables. C'est à ces ajustements entre des dispositions et des contextes dans lesquels s'inscrivent les dispositifs participatifs que font référence les notions de participations dites ordinaires et extraordinaires. Cette recherche s'inscrit dans un questionnement plus large sur l'hypothèse de la demande sociale de participation, sa construction et sa prise en compte par les dispositifs participatifs. De là, interroger une offre de participation comme une opportunité saisie ou non par des citoyens, permet d'accéder à une gamme contextualisée de réponses à la question : comment les individus souhaitent-ils ou sont-ils en mesure de souhaiter participer à la vie démocratique? 


\section{Introduction}

Si les offres institutionnelles de participation renvoient à des réalités diverses que peut révéler l'analyse de leur conception et de leur mise en œuvre, cela est également le cas des appropriations qu'en ont ceux qui y prennent part. Des appropriations qui sont socialement différenciées et qui s'articulent aux logiques et aux ressources mobilisables et mobilisées dans la participation. Notre hypothèse est ainsi celle d'appropriations différenciées d'une offre institutionnelle de participation s'expliquant à la fois par l'offre et par la demande, à travers les modalités de participation mises en œuvre et les positions sociales et trajectoires des participants. En effet, si les politiques de participation publiques doivent se comprendre comme des politiques de l'offre, elles ne s'imposent pas pour autant de manière homogène à la population et à ceux qui y prennent part, mais s'inscrivent dans un contexte et dans une rencontre avec des pratiques préexistantes. L'attention duale aux effets d'offres et à leurs appropriations par des participants localement et socialement situés permet de rendre compte des investissements différenciés dans les dispositifs participatifs, d'une manière que ne peut faire une approche unilatéralement centrée sur les caractéristiques sociales des participants ou sur les acteurs de l'offre.

À travers les réponses faites à une proposition de participation, c'est la question des conditions de l'attrait d'une offre de participation qui est posée. Ainsi, interroger les tenants et aboutissants d'une offre institutionnelle de participation publique amène à remonter aux raisons qui la fondent, à la fois pour ceux qui la font et pour ceux qui y vont. En ce sens apparaît de manière éparse dans la littérature le terme de " demande sociale de participation » pour désigner et questionner l'idée d'une demande de la population à laquelle une offre de participation viendrait répondre, ou du moins dans laquelle des acteurs investis en tant qu'offreurs de participation trouveraient une justification. Dans le même temps, davantage qu'une demande sociale de participation que les différents dispositifs participatifs viseraient à absorber ou à canaliser, ressort de l'observation l'hypothèse d'une demande sociale par la participation, où l'enjeu de la mobilisation de citoyens désintéressés devient crucial en tant que moyen d'asseoir la

[1] La « demande sociale de participation » est évoquée comme « mise en avant [par les associations] pour réclamer la mise en place d'une démocratie plus participative » (Blatrix, 2001, p. 303-304) et par là comme une création des procédures participatives [contribuant] à faire advenir ce qui les justifie » (Blatrix, 2000, p. 63). Estimée « incertaine et faible», elle renvoie à « [l'intérêt de] nombreux sociologues [pour] le désir social de participation dans les années 1960 et 1970 » (Paoletti, 1997, p. 50). Elle est intrinsèquement liée à la« mobilisation de la thématique [participative] qui s'inscrit dans la volonté de renouveler les pratiques politiques, de répondre à une demande sociale de participation (dont il est permis de douter) » (Lefebvre, 2007, p. 21) et à des dispositifs pensés comme « des technologies d'ajustement à la demande sociale» (ibid.). Enfin, elle se justifie dans la conception d'un pouvoir « maintenant perçu comme légitime que s'il se soumet régulièrement à une épreuve de discussion et de justification » (Rosanvallon, 2006, p. 302), mais alors elle n'est pas prise directement pour objet, mais convoquée sous une forme tautologique à travers l'affirmation que « la démocratie participative correspond d'abord à une demande sociale » (ibid.). 
légitimité des processus participatifs. C'est cette perspective de la participation publique comme politique de l'offre (Gourgues, 2012) qui rend possible la déconnexion entre offre et demande. L'enjeu de l'offre n'est alors pas tant de répondre à une demande de démocratisation que de la promouvoir et, par là, de justifier son existence et sa pertinence. La demande serait alors constituée par l'offre en tant que telle, et ce qui serait alors à l'œuvre serait «la construction sociale de ce qui est donné à voir comme politique publique, susceptible de faire figure de réponse à une demande » (Anquetin, Freyermuth, 2008, p. 11).

Notre démarche empirique vise à une meilleure compréhension des dispositifs participatifs dans leur environnement (Carrel, 2009) en interaction avec ce qui leur préexiste, à l'échelle du territoire et des trajectoires des individus. Il s'agit de contextualiser les innovations participatives dans un ordinaire de l'engagement local et d'étudier la variété des publics, leurs insertions dans des systèmes d'interconnaissance et de reconnaissance et leurs motifs d'engagements participatifs. Qui sont les participants et comment la participation s'inscrit-elle dans leur trajectoire? Qu'est-ce que cela implique en retour pour l'offre de participation? Et comment de là apparaissent en creux les conditions d'émergence d'une participation qui sortirait de l'ordinaire auquel s'arriment les dispositifs ?

Le présent développement s'appuie principalement sur l'observation d'une offre publique de participation à l'échelle d'une municipalité de 17000 habitants mise en œuvre entre 2008 et 2014. Les données utilisées ont principalement été recueillies par entretiens et questionnaires auto-administrés, et constituent le premier terrain d'une recherche doctorale en cours. Des observations ont également été menées ${ }^{2}$ sans que le matériau qui en est issu ne soit directement mobilisé ici. Les entretiens ont été réalisés avec deux élus, maire et délégué à la citoyenneté, une fonctionnaire en charge de cette politique et 24 participants à des dispositifs participatifs entre l'été 2012 et l'hiver 2013. Nous avons envoyé 275 questionnaires fin 2012 ; ces 275 individus correspondent à l'ensemble de la population ayant pris part à un dispositif participatif ${ }^{3} .101$ retours ont été recueillis, soit $37 \%$. Les participants rencontrés en entretien ont été sélectionnés parmi les répondants pour refléter la diversité de l'échantillon et permettre d'approfondir l'analyse, et notamment les différents parcours mis en évidence.

Le questionnaire interroge les caractéristiques socio-économico-démographiques des répondants, leurs rapports à la politique, à la vie locale et à

[2] Forum citoyen en novembre 2012, réunion de comité d'usagers d'un espace culturel en novembre 2013 et participation à un cycle de formation agents-élus sur la démocratie locale en juin 2012.

[3] Pour réaliser cette liste, nous avons compilé deux principales sources : un fichier fourni par les services de personnes ayant «participé à une ou plusieurs démarches de démocratie locale » et la liste d'émargement du forum citoyen de novembre 2012. Ces sources ont été complétées à la marge en recoupant les noms figurant dans les comptes rendus disponibles en ligne lforums, groupes de travail) avec l'annuaire. 
l'engagement. Ces éléments sont mis en perspective avec différentes informations sur leur participation à un ou des dispositifs participatifs et un questionnement quant aux «motivations » de leur participation ${ }^{4}$. Le questionnement fermé sur ces motivations, en tant que motifs d'engagement dans les dispositifs participatifs, s'appuie sur des entretiens exploratoires et sur la littérature sur l'engagement participatif, notamment les quatre motifs répertoriés par A. Mazeaud et J. Talpin (2010) : civisme, sociabilité, enrichissement cognitif, intérêt personnel. Nous avons retenu huit propositions s'y rattachant : curiosité pour la démarche participative, intérêt pour le sujet, faire valoir un point de vue personnel, apprendre des choses, rencontrer des gens, faire de la politique, mieux connaître les élus, améliorer les politiques publiques. L'analyse des questions ouvertes nous en fait ajouter huit autres : comprendre le fonctionnement d'une ville, s'intégrer dans la commune, se rendre utile, faire bouger les choses, jouer un rôle citoyen actif, améliorer son cadre de vie, donner son avis, répondre à une invitation.

Il s'agit à travers ces données d'analyser des registres de justification et d'appréciation de la participation, en les croisant avec les différents types de dispositifs, les propriétés sociales et les pratiques déclarées des individus. Autrement dit, rejoignant l'hypothèse de participants qui « s'engagent dans les dispositifs selon différentes figures de participation » (Richard-Ferroudji, 2011, p. 164), ce que nous nommons ici appropriations différenciées, il s'agit de «questionner de manière croisée les dispositifs et les dispositions à participer » (p. 165). En effet, notre terrain se caractérise par ce que nous nommons une gamme d'opportunités de participation, permettant de « développer l'analyse comparative de différents formats de participation » et d'esquisser une « analyse comparée de la variété des offres d'engagement [et] poser les jalons d'une sociologie de la participation sensible à la pluralité et la variabilité des formes d'engagement »(Mazeaud, Talpin, 2010, p. 372), pour reprendre leur conclusion en forme de programme de recherche à la question de «Participer, pour quoi faire?».

Notre recherche, dans laquelle s'insère la présente analyse, en partant de l'offre pour s'intéresser aux trajectoires qu'elle rencontre, se positionne comme une illustration empirique de la controverse autour de la notion de demande sociale

[4] La notion de « motivation » ne va pas sans poser de difficultés. En effet, comme le pointent M. Crozier et E. Friedberg : «Cette logique des motivations [...] est partielle et pauvre. [...] Partielle, donc peu efficace, parce qu'elle ne peut tenir compte du fait que l'homme découvre ses désirs en fonction des opportunités qu'il aperçoit. Pauvre, parce qu'elle réduit l'homme à la décision par laquelle il s'exprime, alors que ce choix, toujours forcé, n'est jamais qu'une des virtualités possibles dans laquelle effectivement il s'aliène souvent, mais tout de même pas nécessairement » (1977, p. 368). Dans notre emploi de ce terme, nous prenons la précaution de rappeler la portée compréhensive de notre démarche et l'appréciation forcément rétrospective qui est donnée à cette notion par des acteurs interrogés a posteriori. Ainsi, elles sont réinterprétées dans l'analyse pour une part en « motifs » ou « justifications » d'actions qui sont fonction des opportunités, ici l'offre de participation à laquelle ils ont répondu. Interroger ce qui « a motivé à participer » est autant un emprunt au langage indigène qu'une facilité de langage dans la relation enquêteur-enquêtés. 
de participation. En effet, à travers différentes recherches sur la participation, issues notamment de la sociologie étatsunienne, s'est constitué un débat qui a pu être interprété comme portant sur la demande sociale de participation ${ }^{5}$. Les résultats de J. Hibbing et $\mathrm{E}$. Thiess-Morse et leur thèse de la stealth democracy (2002) sont souvent rappelés comme la source de cette controverse, notamment par ceux qui entendent y répondre pour approfondir ou atténuer les conclusions qu'elle charrie ${ }^{6}$. Ce caractère furtif de la démocratie implique ainsi pour la démocratie participative qu'il n'existe pas de «demande », comme le résument G. Gourgues et J. Sainty (2011) lorsqu'ils expérimentent l'importation de ces questionnements dans ce qu'ils nomment « le monde réel de la participation ». Une des limites, qui leur sert également de conclusion, étant de tester cette réalité par la mesure par sondage de la connaissance du grand public des dispositifs participatifs, plutôt que sur la compréhension des usages et des pratiques qui donnent corps à la participation, à défaut d'en faire « un phénomène politique suffisamment significatif au sein du "grand public" pour faire l'objet d'une enquête de ce type » (2011, p. 22).

L'étude d'une offre institutionnelle municipale et de ses appropriations différenciées se veut un apport à la compréhension des logiques et des conditions d'une « demande de participation » au regard du positionnement de l'offre. En effet, qu'elle soit réinterprétée à travers les enjeux académiques, politiques ou marchands liés aux dispositifs participatifs, la question de la demande de participation reste une déclinaison d'un questionnement classique de la science politique : comment les personnes souhaitent-elles, ou sont-elles en mesure de souhaiter, participer à la vie démocratique ? Interroger une offre de participation comme une opportunité saisie ou non par des citoyens qui, ce faisant, accèdent pour une part au statut de participants à des degrés divers et suivant des logiques différenciées, c'est avoir accès à une gamme contextualisée de réponses à cette question et comprendre comment la participation s'inscrit dans des trajectoires.

[5] Voir Gourgues, Rui, Topçu (2013, p. 14).

[6] La controverse autour de la demande de participation articule remise en cause au profit d'une démocratie «furtive » (stealth) (Hibbing, Thiess-Morse, 2002, 2005), signe des manquements du gouvernement représentatif plus que d'une demande (Bowler et al., 2007) et volonté ne pouvant se concrétiser faute d'une offre exigeante et convaincante (Neblo et al., 2010). Plus récemment, des développements ont été apportés par P. Webb (2012) distinguant «stealth » and «dissatisfied » democrats, les seconds étant majoritaires, mais à la participation latente faute d'offre convaincante, et par des recherches proposant à partir de ces résultats de tester les correspondances entre les parcours de participation politique et l'expression de préférences procédurales représentatives, technocratiques ou participatives (Bengtsson, Christensen, 2013). 


\section{La démocratie locale par les dispositifs participatifs : pour quoi et avec qui ?}

«De nombreux habitants nous ont dit leurs attentes, leurs besoins, leurs envies. Le dialogue a commencé et sera demain la base des relations que nous établirons avec vous [...] nous nous y engageons, [il] ne s'arrêtera pas au soir des élections » (Profession de foi, 2008)

"La démocratie locale est une volonté politique affichée, et répétée, du maire et de son équipe. [...] Le maire parlait même de "spécificité": "Une ville, c'est d'abord des habitants. Depuis quatre ans, on conçoit toutes nos actions en les impliquant dans le processus." [...] D'autres communes de la Métropole s'inscrivent dans cette démarche, mais "la ville est un peu réputée comme un laboratoire d'expériences" » (Ouest-France, 2 novembre 2012)

\section{Une offre de participation inscrite dans l'inédit de l'alternance}

Entre 2008 et 2014, une liste de gauche plurielle composée principalement de militants associatifs a accédé au pouvoir dans la deuxième ville de la communauté d'agglomération rennaise. Depuis la campagne jusqu'au mandat, cette équipe s'est fortement appuyée sur la rhétorique et la mise en œuvre de politiques de participation publique se matérialisant dans une série de dispositifs participatifs. Ce regroupement a démarré à la fin des années 1990, avec la création d'une association « pour réfléchir ensemble à la manière dont les habitants pouvaient prendre la parole et s'organiser pour donner leur avis, [à un] moment où personne [ne] leur demandait ${ }^{7}$. Suite à une défaite au premier tour en 2001 , face au maire se représentant pour un troisième mandat, une autre association, « Cap à gauche », est créée. Elle regroupe entre 60 et 80 personnes et sert de structure pour préparer la prochaine échéance électorale et la prise de rôle de ces futurs élus « pas habituels, qui viennent du monde associatif, militant ${ }^{8}$.

Pourtant au-delà du rappel d'une campagne participative, qui fait office de récit fondateur pour les élus et les membres encore actifs de l'association, l'élection doit beaucoup au jeu politique local, du fait de la division de l'ancienne majorité de droite? . Dès lors, ce récit agit avant tout comme un énoncé politique qui vient

[7] Entretien, maire.

[8] Entretien, fonctionnaire en charge de la démocratie locale.

[9] Ouest-France (17 mars 2008) publie des réactions postélectorales qui résument cette configuration unique pour la commune : "C'est un moment fort. Jusqu'à présent, la gauche [...] n'était majoritaire que lors des élections nationales » (ancienne tête de liste socialiste) ; " Je pensais que c'était jouable, mais pas à ce point-là. On estime, en fait, qu'entre un tiers et la moitié des électeurs de [la liste de droite dissidente] ont voté pour notre liste » (tête de liste vainqueur) ; «Dans l'union, nous aurions dû gagner dès le premier tour. Si l'UMP et le Modem avaient été honnêtes avec eux-mêmes, ils [nous] auraient soutenu » (ancien maire). 
donner du sens à une élection en partie inattendue. L'interprétation d'une dynamique de campagne au travers du prisme participatif lquestionnaire, réunions publiques, réunions chez l'habitant...) se veut la préfiguration et l'incarnation d'un mode opératoire, illustré par la suite à travers les dispositifs participatifs. Ceux-ci s'inscrivent ainsi dans une «fabrique de l'alternance » (Mazeaud, 2010), un « activisme symbolique » (Lefebvre, 2012) et une remise en cause de l'ancienne figure mayorale de la proximité. Un « maire qui disait "moi je connais tous les habitants" [alors qu'eux] au contraire [ont] conscience d'une diversité de situations [et se posent] toujours la question de voir qui sont les habitants, les citoyens et les électeurs $\gg^{10}$. Dans le contexte d'une ville dont le nombre d'habitants a doublé depuis 1990 et en observant que « plus la commune est grande, plus la municipalité se focalise sur la démocratie participative, comme moyen de se rapprocher des électeurs » (Premat, 2009, p. 12), l'implantation de dispositifs participatifs, comme mode de gestion municipale et marque d'un changement de méthode et de style politique, renvoie à un enjeu de distinction par rapport à l'opposition. La démarche de participation citoyenne donne une assise aux élus d'une majorité en partie dans une phase d'apprentissage du champ politique local. Mais le choix de fonder ainsi le projet municipal est aussi une potentielle fragilité, vis-à-vis d'une partie de la population, soit qui serait déçue du caractère uniquement consultatif, soit qui ne se retrouverait pas dans «toutes [leurs] discussions [qui ont fait] perdre du temps et l'argent $\gg^{11}$. La gestion des contradictions par la participation rend possible une critique à double sens : elle peut dans le même temps n'être pas encore assez et être déjà de trop. Se pose alors la question de qui sont les publics se trouvant impliqués à des degrés divers dans cette démocratie locale?

\section{L’offre et ses publics : prime d'ancienneté et sélectivité sociale}

Notre échantillon, représentant un peu plus d'un tiers des «participants », rappelle et confirme dans ses grandes composantes les constats sur la sélectivité sociale des dispositifs participatifs. Il est essentiellement masculin, et âgé de 40 à 60 ans. Les groupes d'âge particulièrement peu présents sont les moins de 30 ans et les plus de 70 ans. Les femmes sont sous-représentées, seules les 41-50 ans sont présentes dans une proportion comparable aux hommes. Les cadres et professions intellectuelles supérieures et les retraités sont surreprésentés par rapport à leur poids dans la population municipale. Ces deux groupes représentent $62 \%$ des 101 répondants. Les catégories intermédiaires et sans activité professionnelle qui apparaissent occultées au regard de leur poids dans la population municipale. Sept répondants se déclarent ouvriers, mais affirment en même temps des particularismes laujourd'hui retraités ; secrétaire régional, délégué fédéral d'un syndicat ; au chômage ; employé titulaire d'un bac technologique), qui soulignent la forte spécificité des membres des catégories populaires qui prennent part à la

[10] Entretien, maire.

[11] Inquiétude d'un élu après des actes de vandalisme commis sur un abri réalisé avec et pour des jeunes de la ville, et pas toujours bien accueilli par les riverains. 
démocratie locale et qui constituent sous divers aspects une double exception, à la fois au sein de l'ensemble des participants et de leur catégorie professionnelle.

Au sein de notre échantillon, $28 \%$ s'apparentent aux classes populaires ${ }^{12}$. Sans tenir compte de la composition du foyer, $44 \%$ ont un revenu mensuel net inférieur à $3000 €$, parmi eux $46 \%$ n'ont pris part à aucun ${ }^{13}(15 \%)$ ou à un (32\%) type de dispositif, et $54 \%$ à deux (27 \%) ou trois ou plus (27\%). Tandis que ceux ayant un revenu supérieur à $3000 €$ sont $27 \%$ à n'avoir pris part à aucun (8\%) ou à un type de dispositif (19\%) et $74 \%$ à avoir pris part à deux (34\%) ou trois ou plus (40\%). Les écarts de revenus sont ainsi corrélés à l'intensité et la diversité de l'engagement participatif dans différents types de dispositifs. Si ce constat de la mesure de l'intensité de la participation en fonction du revenu est parlant, il peut aussi être nuancé. L'effet du revenu est ainsi atténué en partie par la prise en compte de l'ancienneté de résidence.

La répartition entre les $37 \%$ de l'échantillon résidant dans la commune depuis moins de 10 ans, et les $64 \%$ y résidant depuis plus de 10 ans, illustre ainsi une présence de plus en plus forte des seconds selon le nombre de dispositifs cités. Les participations les plus intenses et diversifiées en termes de nombre de types de dispositifs fréquentés concernent avant tout une majorité d'anciens habitants, nonobstant le seuil de revenu. Il apparaît ainsi dans la mesure de la durée de résidence dans la commune une tendance rectificative de l'effet des écarts de revenus par rapport à l'intensité de la participation, indice potentiel d'une forme de « capital d'autochtonie » (Retière, 2003), mais qui redouble en retour la faible présence des plus faibles revenus arrivés récemment.

Tableau 1. Intensité de la participation, selon revenus mensuels nets par foyer et ancienneté de résidence

\begin{tabular}{|c|c|c|c|c|c|c|}
\hline \multicolumn{2}{|c|}{ Participation à « $\mathrm{x} »$ types de dispositifs } & 0 & 1 & 2 & 3 et plus & $\begin{array}{c}\% \text { de } \\
\text { l'ensemble } \\
\quad[n=94]\end{array}$ \\
\hline \multicolumn{2}{|c|}{ Moins de $3000(n=41)$} & $15 \%(6)$ & $32 \%(13)$ & $27 \%(11)$ & $27 \%(11)$ & $44 \%$ \\
\hline \multirow[t]{2}{*}{... dont résidents } & depuis plus de 10 ans & (2) & (8) & (9) & (10) & $31 \%(29)$ \\
\hline & depuis moins de 10 ans & (4) & (5) & (2) & (1) & $13 \%(12)$ \\
\hline \multicolumn{2}{|c|}{ Plus de $3000(n=53)$} & $8 \%(4)$ & $19 \%(10)$ & $34 \%(18)$ & $40 \%(21)$ & $56 \%$ \\
\hline \multirow[t]{2}{*}{... dont résidents } & depuis plus de 10 ans & (2) & (4) & (13) & (15) & $36 \%(34)$ \\
\hline & depuis moins de 10 ans & (2) & (6) & (5) & (6) & $20 \%(19)$ \\
\hline
\end{tabular}

[12] En prenant comme seuil de revenu mensuel net moins de $1120 €$ pour un foyer d'une personne, moins de $2240 €$ pour un foyer de deux et moins de $3000 €$ pour un foyer de 3 et plus (cf. Observatoire des inégalités : http://www.inegalites.fr/).

[13] La question concerne la participation à un type de dispositifs mis en œuvre (cf. tableau 3), en dehors du forum annuel, d'où la possibilité de répondre aucun, et sans prendre ici en compte la possibilité d'avoir participé à plusieurs dispositifs du même type. 
Enfin, les participants apparaissent comme fortement diplômés. La catégorie des $\mathrm{Bac}+4 / 5$ représente un quart de notre échantillon, et celle du premier cycle universitaire est surreprésentée à hauteur de $+17 \%$ par rapport aux données du recensement sur l'ensemble de la population municipale. Par rapport à la mesure de l'ancienneté, les deux sous-ensembles de notre échantillon ayant un niveau de diplôme inférieur au Bac ou entre le Bac et Bac +2 représentent chacun $30 \%$ de l'ensemble, et comptent environ deux tiers de résidents depuis plus de 10 ans, tandis que la catégorie des Bac +3 à Bac +5 représente $40 \%$ et comprend un peu plus de la moitié d'anciens. Si l'ensemble est surdiplômé, il y a en son sein autant d'anciens en deçà du baccalauréat que de nouveaux ayant suivi entre trois et cinq ans un cycle universitaire, les premiers ayant même une intensité de participation légèrement plus élevée. Comme pour le revenu, les catégories les moins diplômées ayant une forte participation se recrutent exclusivement parmi les anciens habitants.

Tableau 2. Intensité de la participation, selon diplôme et ancienneté de résidence

\begin{tabular}{|c|c|c|c|c|c|c|}
\hline $\begin{array}{l}\text { Niveau de } \\
\text { diplôme }\end{array}$ & $\begin{array}{c}\text { Résidant } \\
\text { depuis plus } \\
\text { de } 10 \text { ans }\end{array}$ & $\begin{array}{c}\text { Participa } \\
0\end{array}$ & $\begin{array}{c}\text { ion à } \ll x ~ \\
1\end{array}$ & $\begin{array}{c}\text { types de } \\
2\end{array}$ & $\begin{array}{c}\text { lispositifs } \\
3+\end{array}$ & $\begin{array}{c}\% \text { de } \\
\text { l'ensemble } \\
\text { (n=101) }\end{array}$ \\
\hline \multirow{2}{*}{$\begin{array}{l}<B a c \\
(n=29)\end{array}$} & OUI & $5 \%(1)$ & $26 \%(5)$ & $26 \%(5)$ & $42 \%(8)$ & $19 \%$ \\
\hline & NON & $40 \%(4)$ & $30 \%(3)$ & $30 \%(3)$ & 0 & $10 \%$ \\
\hline \multirow{2}{*}{$\begin{array}{c}\text { Bac à Bac }+2 \\
(n=31)\end{array}$} & OUI & $9 \%(2)$ & $18 \%(4)$ & $36 \%(8)$ & $36 \%(8)$ & $22 \%$ \\
\hline & NON & $11 \%(1)$ & $56 \%(5)$ & $22 \%(2)$ & $11 \%(1)$ & $9 \%$ \\
\hline \multirow{2}{*}{$\begin{array}{c}B a c+3 \text { à } B a c+5 \\
(n=41)\end{array}$} & OUI & $4 \%(1)$ & $13 \%(3)$ & $39 \%$ (9) & $43 \%(10)$ & $23 \%$ \\
\hline & NON & $11 \%(2)$ & $22 \%(4)$ & $28 \%(5)$ & $39 \%(7)$ & $18 \%$ \\
\hline
\end{tabular}

Les participants à la démocratie locale sont ainsi d'abord marqués par une ancienneté de résidence. De là, les différents sous-ensembles sont ordonnés en relation avec le niveau de diplôme et de revenu. Une tentative de hiérarchisation des marqueurs de la participation à la démocratie locale compterait ainsi avant tout l'ancienneté, complétée par le niveau de diplôme et de revenu, en tout cas au-delà d'un certain seuil impliquant l'absence des classes les plus populaires.

Ce rappel général est nécessaire pour éviter que l'étude des appropriations différenciées occulte cette donnée première de la sélectivité des dispositifs participatifs. C'est d'ailleurs même ce constat, connu autant de la littérature que des promoteurs de l'offre, qui appelle en partie l'intérêt pour la mise en forme d'une offre de participation sous forme de «gamme d'opportunités ». 


\title{
La mise en forme d'une gamme d'opportunités de participation
}

\begin{abstract}
«Dans nos instances thématiques, il y a des personnes qu'on voit tout le temps, je pense que c'est inévitable, mais il y a vraiment à chaque fois des nouvelles personnes, dans chaque groupe... et des personnes différentes. Parce qu'on varie les thèmes et il n'y a pas du tout la même population dans le comité sur les aires de jeux que dans le groupe mémoire, par exemple. Donc je pense qu'on a beaucoup plus de diversité qu'en faisant simplement des conseils de quartier. » Fonctionnaire en charge de la démocratie locale, Entretien
\end{abstract}

La participation des citoyens est décrite dans la communication de la municipalité comme relevant de la démocratie locale, un terme englobant « l'ensemble des dispositifs pour que chacun trouve sa place $\gg^{14}$ et qui sont vus comme permettant de viser au plus large en termes de mobilisation, mais aussi comme s'imbriquant potentiellement entre eux. Le choix a été fait de décliner l'offre sous l'angle thématique plutôt que de mettre en œuvre des instances territoriales pérennes de type conseils de quartier. Ce qui est à l'œuvre, c'est la conception d'une offre se voulant large et diversifiée, pour rencontrer un public répondant aux mêmes qualificatifs. Cette orientation du projet municipal s'est traduite dès le début du mandat par des dispositifs ponctuels et thématiques, classés en cinq catégories : réunion publique, rendez-vous de proximité, comité consultatif, atelier thématique, comité d'usagers et par la tenue annuelle d'un forum.

La notion de gamme incite à des appropriations différenciées, qui s'expliquent donc potentiellement par les caractéristiques sociologiques, dispositions et trajectoires des participants, mais aussi comme une intentionnalité de l'offre. La mise en offre de la participation est marquée par une forte réflexivité quant au cadre méthodologique de la part des élus, notamment le maire « initiateur [...] [et qui s'il] n'avait pas été élu, aurait travaillé dans ce domaine-là $\gg^{15}$ et l'adjoint délégué « un ami de longue date, [qui] dans l'équipe était le plus proche, de [cette] sensibilité $\gg^{16}$. En écho à cette préoccupation de prise en charge de « l'impensé procédural » (Blondiaux, 2004) s'est développée une mise en récit de l'évolution des dispositifs participatifs. Un forum organisé en 2012 a constitué le « véritable tournant », la « charnière » entre l'expérimentation et la mise en cohérence. Les modalités de participation ont été mises en pratique dès 2009 , mais formalisées en 2012 dans un « mode d'emploi de la démocratie locale » pour communiquer en externe et s'organiser en interne dans les services avec un plan d'action annuel et des fiches de cadrage des dispositifs.

[14] Entretien, maire.

[15] La détention du Diplôme d'État relatif aux Fonctions d'Animateur (DEFA) et ses expériences professionnelles (directeur d'un organisme de formation, d'un centre d'information jeunesse puis d'un organisme d'action et d'animation socialel évoquent ainsi un profil proche de certains professionnels de la participation.

[16] Entretien, maire. 


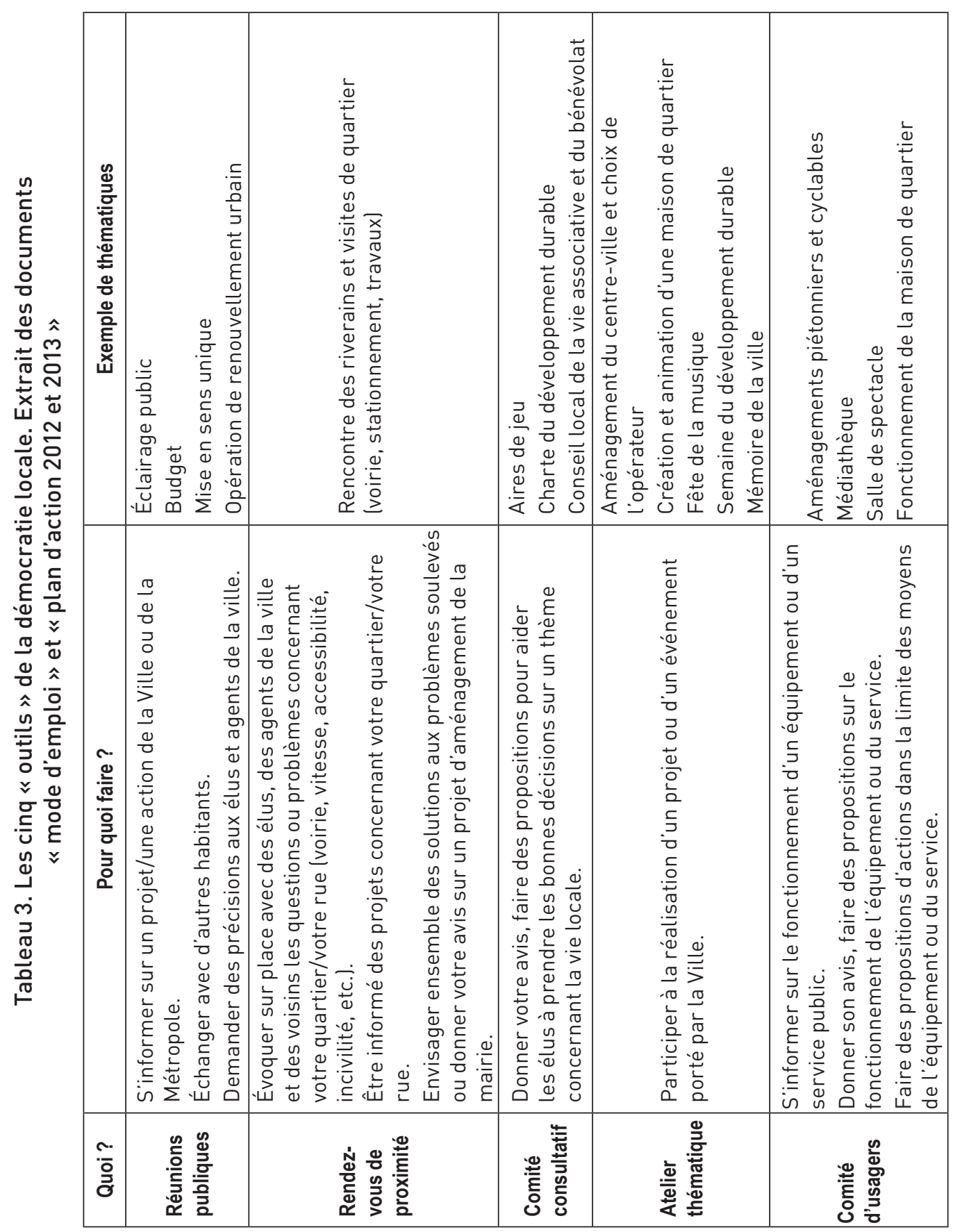


À ce cadre méthodologique, sont adjointes plusieurs remarques de la municipalité qui dessinent les contours d'une forme de contrat avec les participants et indiquent la portée et les limites données à l'exercice auquel ils sont invités à prendre part. Ainsi, «Pour que ça marche, la mairie s'engage à » :

Tableau 4. Le contrat « d'écoute sélective transparente ». Extrait du document « mode d'emploi »

\begin{tabular}{|l|l|}
\hline $\begin{array}{l}\text { Viser l'intérêt } \\
\text { général }\end{array}$ & $\begin{array}{l}\text { Participer pour défendre un intérêt personnel, c'est légitime et souvent } \\
\text { constructif. Mais les dispositifs mis en place par la Ville sont au ser- } \\
\text { vice de tous : il faut savoir respecter l'avis des autres et accepter que } \\
\text { la décision prise ne soit pas forcément celle que l'on attendait. }\end{array}$ \\
\hline $\begin{array}{l}\text { Permettre } \\
\text { l'expression } \\
\text { du plus grand } \\
\text { nombre }\end{array}$ & $\begin{array}{l}\text { Permettre à tous de participer, c'est aussi adapter les outils aux } \\
\text { attentes et aux contraintes des habitants : varier les thèmes et les } \\
\text { niveaux d'implication, travailler sur des sujets concrets, imaginer de } \\
\text { nouvelles formes de participation comme les rencontres sur place, les } \\
\text { tirages au sort, les enquêtes, les sondages via internet... }\end{array}$ \\
\hline $\begin{array}{l}\text { Être clair } \\
\text { et précis }\end{array}$ & $\begin{array}{l}\text { Un plan d'action annuel définit les sujets et projets pour lesquels un } \\
\text { dispositif de participation est mis en place. Ce plan fait l'objet d'une } \\
\text { présentation et d'un vote en Conseil municipal. Une fiche de cadrage } \\
\text { (calendrier, objectifs, fonctionnement, budget, contraintes juridiques } \\
\text { et techniques...) est diffusée aux participants lors de chaque nouvelle } \\
\text { démarche. }\end{array}$ \\
\hline $\begin{array}{l}\text { Rendre } \\
\text { compte du } \\
\text { résultat et } \\
\text { des suites } \\
\text { données }\end{array}$ & $\begin{array}{l}\text { Point sur les suites données aux propositions faites lors de la réunion } \\
\text { précédente. } \\
\text { Bilan annuel de l'ensemble des démarches de participation. }\end{array}$ \\
\hline
\end{tabular}

Ce document résume le cadre donné à la participation des habitants et illustre l'application dans le discours d'une collectivité locale de la notion « d'écoute sélective transparente » (Talpin, Sintomer, 2011) pour décrire l'usage et la portée des dispositifs participatifs, et entérine le choix d'une « gamme » d'opportunités de participation, plutôt qu'un fonctionnement par instances pérennes, pour « permettre l'expression du plus grand nombre ». 
Cette formalisation de l'offre se reflète dans l'évolution du «forum de la démocratie locale », devenu en 2012 « forum citoyen ». Ce dispositif événementiel, qui a aussi une fonction de vitrine, a d'abord été positionné par les élus comme une réflexion autour de la mise en œuvre de la démocratie participative. Ainsi, durant ces trois premières éditions de 2009 à 2011, le programme est centré sur un propos théorique et méthodologique ${ }^{17}$ pour ensuite s'orienter plus explicitement vers la mise en discussion de politiques sectorielles en 2012 et être renommé à ce moment «forum citoyen », avec propositions et vote des citoyens, puis retour détaillé de la municipalité dans un document écrit. Il est annoncé le passage d'un ordre du jour procédural à substantiel : « le processus rentre dans une phase plus active [...] il n'est plus question seulement de principe et de méthodologie, mais d'avis et de propositions $\gg^{18}$.

\section{L'exemple du forum 2012 et l'enjeu d'une meilleure inclusion}

Nous nous concentrons ici sur les évolutions du forum annuel. Parmi notre échantillon de 101 répondants, nous avons pu recueillir les réponses de $70 \%$ des 89 habitants présents à l'édition 2012 du forum, qui a réuni 118 personnes, élus, agents de la ville et invités extérieurs inclus. Considérant la présence au forum de 2012 et aux autres éditions, quatre groupes se dégagent parmi les répondants, pour interroger si le public du « forum citoyen » de 2012 est tendanciellement différent du public du « forum de la démocratie locale » des années précédentes.

Tableau 5. Répartition des 101 répondants en fonction de la participation au forum de 2012

\begin{tabular}{|c|c|c|c|}
\hline \multicolumn{2}{|r|}{ Groupes } & $n=$ & Définition \\
\hline 1 & Non-participants au forum & 16 & Aucun forum. Ont pris part à d'autres dispositifs \\
\hline 2 & Nouveaux venus & 37 & Uniquement forum de 2012 \\
\hline 3 & Participants absents en 2012 & 22 & 1 ou 2 forums, en dehors de 2012 \\
\hline 4 & $\begin{array}{l}\text { Participants réguliers } \\
\text { présents en } 2012\end{array}$ & 26 & 2 forums (14) et 3 ou 4 forums (12), dont 2012 \\
\hline
\end{tabular}

[17] Respectivement : «Impératif participatif et légitimité des élus », « Comment prendre en compte la parole des habitants?», «Des outils pour agir ensemble».

[18] Interview du délégué à la citoyenneté, Ouest-France, 30 octobre, 2 novembre 2012. 
En 2012, Ouest-France pointe que «la majorité des inscrits vient pour la première fois ». Ce raccourci journalistique comporte une part de vérité. En effet, le groupe 2 tend à une plus grande inclusion des catégories les moins présentes dans l'ensemble de l'échantillon. Les catégories professionnelles et les niveaux de diplôme surreprésentés se répartissent dans l'ensemble des groupes, le 2 y compris, et sont prépondérants parmi les participants « réguliers ». Mais les catégories en sous-poids dans notre échantillon sont concentrées dans le groupe 2 : 5 ouvriers sur $7 ; 4$ personnes sans activité sur 6 ; 8 BEP-CAP sur 13 . Cette tendance se trouve confirmée en observant la répartition des niveaux de revenus mensuels des foyers entre ces cinq groupes : 4 sur 4 des revenus inférieurs à $1120 €$ et 9 sur 20 de la tranche 1 120-2 $240 €$ se trouvent parmi les nouveaux venus. De plus, le groupe 2 présente la deuxième plus élevée des mesures d'écarts-types de revenu, signe d'une relative hétérogénéité.

Les faibles effectifs n'autorisent aucune généralisation, mais il apparaît que le groupe 2 semble plus inclusif socialement que les autres. Autrement dit : le peu de représentants des classes populaires que comporte notre échantillon ont tendanciellement acquis leur qualité de participants via leur présence au forum citoyen de 2012, plutôt qu'aux précédents ou en dehors de ces forums. Cet élargissement de la participation s'observe aussi à travers la variable territoriale : parmi les 15 habitants d'un nouveau quartier que compte notre échantillon, désignés comme les «éloignés » des dispositifs participatifs dans nos entretiens, 10 appartiennent au groupe 2 et aucun au groupe 4, tandis que les habitants du centre historique sont concentrés dans le groupe des plus assidus. Cet effet peut être attribué symboliquement au repositionnement du forum et à une certaine légitimité acquise par l'inscription dans le temps, et matériellement au recours à un institut régional de sondage pour relancer par téléphone des tirés au sort, cette pratique se limitant auparavant à une invitation écrite, sans logique de quotas. Sur la centaine de participants, un quart est venu suite à ce démarchage ${ }^{19}$. Il y a ici un exemple d'inclusion réelle, et qui par rapport à notre questionnement relève davantage d'un outillage de l'offre que de l'émergence d'une demande. Indice qui invite à un approfondissement de la réflexion sur les effets de cadrage de l'offre, rappelant pour une part que «la supposée demande sociale résulte d'un incessant travail de construction » (Anquetin, Freyermuth, 2008, p. 13), et est donc effectivement influencée par des choix politiques et leurs traductions opérationnelles dans la mise en place des dispositifs. Ce primat de l'offre n'implique pas pour autant qu'elle s'impose de manière homogène à ceux qui y prennent part.

[19] 91 personnes ont été contactées pour obtenir 32 confirmations et 25 présents. 


\section{Représentations et appropriations différenciées de l'offre}

L'étude des publics fait ressortir que la classification socio-économique ne suffit pas à expliquer les ressorts de l'engagement dans les dispositifs participatifs. Elle mesure des tendances sans les expliquer. Un apport théorique complémentaire est mobilisable à travers le concept de capital social, tel que défini par R. Putnam (2000) qui met en avant un cercle vertueux où l'adhésion génère un accroissement de la confiance, qui à son tour favorise la participation. Si la notion a ses limites intrinsèques, du fait de son caractère tautologique, faisant souvent d'elle davantage une variable à expliquer qu'une variable explicative, la référence au capital social peut être utile pour expliquer l'entrée en participation, et son éventuel renouvellement à moyen et court terme, le fait de participer pouvant être en soi porteur de capital social. Cela se justifie d'autant plus après avoir montré l'importance de la durée de résidence dans la commune, le capital d'autochtonie pouvant être une forme socialement et localement située de capital social (Renahy, 2010). La question est alors de savoir si le capital va au capital, soulignant ici la reproduction de logiques préexistantes dans les dispositifs participatifs, ou si l'entrée au capital peut sous certaines conditions être accessible à ceux qui en seraient initialement dépourvus, ou si les deux phénomènes se côtoient.

Nous rejoignons ici en partie des travaux considérant la participation publique sous l'angle de ses effets sur les trajectoires individuelles (Talpin, 2011a, 2011b). Nous entrevoyons que si les participants expriment des représentations communes, notamment autour de la politique et de la citoyenneté, ils le font en ayant des caractéristiques et des dispositions socialement situées, qui impliquent des compréhensions et des appropriations différenciées de l'offre de participation.

\section{Redéfinitions du politique et normes de citoyenneté}

Interrogés sur le sens de leur participation, les répondants s'accordent majoritairement sur l'intérêt pour un sujet, l'apprentissage personnel ou la rencontre avec d'autres, davantage que sur l'amélioration des politiques publiques ou une meilleure connaissance des élus. Parmi toutes les modalités proposées, une seule agrège un désaccord majoritaire : «faire de la politique ». Cette affirmation récurrente recouvre un enjeu de redéfinition de ce qui est entendu par politique, pour en mettre en avant une vision apartisane et non conflictuelle (Blondiaux, 2008b). La politique est discréditée en tant qu'enjeu éloigné pour valoriser l'espace local où elle devient « l'acte citoyen [par lequel] on se sent concerné, impliqué $\gg^{20}$, et en tant que lutte d'intérêts personnels et politiciens pour alors valoriser des notions collectives de «vie de la cité [et] de rencontres

[20] Femme, 46 ans, Cadre, Bac $+4 / 5$. 
des élus avec des citoyens $\gg^{21}$. Interrogés sur l'intérêt qu'ils déclarent porter à la politique, $40 \%$ se déclarent pas du tout (13\%) ou pas vraiment (23\%) intéressés, et $58 \%$ disent qu'ils sont plutôt ( $40 \%)$ ou tout à fait (18\%) intéressés. Les précisions apportées renvoient à la même argumentation : « en tout cas, pas au sens vouloir être élu $\gg^{22}$, « si politique est égale à l'avancée du bien commun $»^{23}$, « la vie en société oui, les arrière-pensées avec un côté partisan et prosélyte, non! $\gg{ }^{24}$ Cette redéfinition à l'œuvre chez les participants appuie l'intérêt méthodologique du couplage des questions fermées et ouvertes afin de permettre une forme d'explicitation du sens donné aux réponses.

Les participants à la démocratie locale mobilisent une vision normée de la citoyenneté. Ces citizenship norms comme « ensemble commun d'attentes quant au rôle du citoyen en politique $»^{25}$ (Dalton, 2008, p. 78) prennent deux formes : duty-based et engaged (Dalton, 2007). La première renvoie à des normes d'ordre social (vote, impôt...), tandis que la seconde intègre la participation à des activités en dehors de l'élection (Bolzendahl, Coffé, 2013). Nous observons dans notre cas davantage une combinaison de ces deux visions, qu'une distinction ou une évolution de la première vers la seconde. Par exemple, l'importance du vote est souvent affirmée, et notre échantillon ne comporte aucun abstentionniste ${ }^{26}$, sans être exclusive de l'importance du débat et de l'implication dans la vie locale. Plus que distribuées distinctement ou en concurrence, citoyennetés « morale » et « engagée » semblent superposées. La participation vient davantage compléter le devoir citoyen, l'actualiser dans une mise en cohérence par la pratique, plutôt que prétendre le supplanter et le dépasser dans l'engagement. Ce même si l'offre de participation est un indice de ces « institutions politiques [qui] sont en phase d'adaptation afin d'accepter et d'encourager ces nouvelles formes [...] qui étendent l'influence de la participation citoyenne [...] en accord avec l'évolution de ces normes de citoyenneté $»^{27}$ (Dalton, 2008, p. 93-94).

C'est davantage le cadrage organisationnel qui valorise un registre dans lequel les participants se retrouvent, plutôt qu'une demande préexistante qui se serait écartée d'une vision morale pour revendiquer une vision engagée de la citoyenneté. Il s'exprime pourtant un sentiment d'extension de la citoyenneté à travers cette possibilité de participation qui s'accorde avec un besoin de « se sentir

[21] Femme, 77 ans, Retraitée-Au foyer, Brevet.

[22] Femme, 46 ans, Formatrice insertion sociale, Bac +2 .

[23] Femme, 67 ans, Retraité-Enseignement, Bac.

[24] Homme, 64 ans, Retraité-Enseignement, Bac +3.

[25] Nous traduisons.

[26] Un examen de la liste d'émargement des élections municipales 2014 a validé l'adéquation entre déclaration et pratique de vote. À partir de notre fichier initial, nous avons pointé aléatoirement les deux tiers des répondants (65 sur 101) et des non-répondants (110 sur 174) à notre questionnaire et mis en évidence un taux de participation de $89 \%$ pour ces deux groupes, à une élection où il fut de $63 \%$.

[27] Nous traduisons. 
citoyen de sa ville, s'exprimer en dehors des périodes électorales $»^{28}$, de $《$ donner pour une fois [son] avis sur les choix politiques (en dehors des élections) ! $\gg{ }^{29}$, de vouloir « se sentir concerné en tant que citoyen $»^{30}$, « ne pas être simplement spectateur ou critique, en tant que citoyen réfléchir, comprendre et apporter [sa] petite pierre à l'édifice ${ }^{31}$, tandis que « trop de gens râlent et ne saisissent jamais l'occasion de prendre la parole quand on la leur donne $»^{32}$.

Au-delà du thème du civisme, la plupart des motivations exprimées se raccrochent à la thématique du local : la ville, le quartier, le voisinage. Ces préoccupations sont transversales dans notre échantillon, mais qualifiées différemment. Les personnes les plus diplômées ont tendance à utiliser des expressions plus actives, «faire bouger ma ville », « participer activement à l'évolution de ma ville » et s'investir dans « l'aménagement de la cité pour le futur [qui est] le rôle majeur du politique local $»^{33}$. Tandis que les moins diplômés sont davantage dans une posture d'information pour « mieux connaître le fonctionnement de la ville, se faire des relations ${ }^{34}$. Ils évoquent plus l'échelle du quartier ou du voisinage, tandis que les autres ne les mentionnent jamais exclusivement. Ce faisant, les participants expriment aussi indirectement leur propre vision d'un périmètre de compétence. Les différents niveaux de langage font transparaître différentes ressources et raccourcis dans l'expression de leur sentiment de compétence politique (Blondiaux, 2007), depuis la projection dans l'avenir et la montée en généralité jusqu'au langage de la proximité et du quotidien : planification urbaine contre troubles de voisinage ; projets contre problèmes. Un écart existe alors entre ceux qui évoquent un « problème bien concret dans [leur] quartier (nuisances sonores) pour essayer de trouver de vraies solutions $\gg^{35}$ et ceux qui resituent cette discussion dans un « environnement [...] largement conditionné par des partenaires extérieurs [comme la] communauté d'agglo ${ }^{36}$. Ce constat n'excluant pas le passage d'un registre à l'autre par la fréquentation des dispositifs : "Ce n'était pas mes motivations de départ, mais je suis contente d'en apprendre plus sur le budget de la commune, les dépenses publiques et de mieux connaître les élus ${ }^{37}$. »

\footnotetext{
[28] Homme, 55 ans, Retraité-Cadre RH, Bac $+4 / 5$.

[29] Femme, 29 ans, Orthophoniste, Bac $+4 / 5$.

[30] Homme, 62 ans, Retraité-Commercial, $\varnothing$.

[31] Homme, 66 ans, Retraité-Responsable d'agence de transport, Bac +3 .

[32] Homme, 52 ans, Professeur des écoles, Bac.

[33] Homme, 55 ans, Retraité-Cadre RH, Bac $+4 / 5$.

[34] Homme, 50 ans, Pré-Retraite-Artisan, CAP-BEP.

[35] Homme, 52 ans, Employé, $\varnothing$.

[36] Femme, 45 ans, Cadre $\mathrm{RH}, \mathrm{Bac}+4 / 5$.

[37] Femme, 29 ans, Orthophoniste, $\mathrm{Bac}+4 / 5$.
} 


\section{L'enracinement et l'intégration}

Comme évoqué, l'ancienneté de résidence est une donnée importante concernant les publics de la démocratie locale. Pour une large part, ils sont d'anciens habitants, résidant dans la ville depuis plus de 10 ans pour $63 \%$ d'entre eux, dont la moitié depuis plus de 20 ans. Un quart est néanmoins arrivé depuis moins de 5 ans. Ces nouveaux arrivants sont marqués par un désir d'intégration lié à cette arrivée récente et une curiosité pour la démarche participative, deux motifs plus régulièrement cités par ces catégories, tandis qu'il y a chez les plus anciens une plus forte propension à mettre en avant les motifs «politiques », qu'il s'agisse « d'améliorer les politiques publiques » ou « de faire de la politique », ce dernier restant majoritairement rejeté, mais tendanciellement moins à mesure que l'ancienneté de résidence augmente.

Cette variable est importante dans le contexte d'une ville qui connaît une forte évolution démographique, de 8000 habitants en 1990 à presque 18000 en 2013. Les entretiens avec les habitants les plus anciens sont parsemés de références à une ville « dédoublée » entre l'ancien bourg et les nouveaux quartiers. L'idée que les dispositifs participatifs peuvent jouer un rôle dans l'intégration des nouveaux habitants est directement prise en compte par l'équipe municipale, comme l'illustre l'évolution du forum en 2012 avec un recours au tirage au sort une attention particulière pour les « nouveaux habitants » comptabilisés à part dans les inscriptions et un atelier dédié à « leur accueil et leur intégration ». Les instances de démocratie locale sont un lieu potentiel d'interconnaissance et d'intégration avec un coût d'accès perçu comme relativement faible en comparaison de l'engagement dans des associations locales.

"Quand on est arrivé ici, moi je connaissais personne. Je mettais ma fille à la garderie, et il y avait un café des parents d'organisé. [...] J'ai fait surtout ça pour rencontrer des gens.

Après, la directrice m'a parlé du forum de la démocratie locale [...] là j'ai participé pour la première fois. [...] C'est un bon remède à l'isolement social. [...] Moi, je suis quelqu'un qui aime travailler, voir du monde. C'est vrai que j'avais une vie plus trépidante avant [mon congé parental]. »

F., cadre lau foyer), Bac $+4 / 5$, moins de 3 ans, Entretien

"Sur une commune qui a une grosse expansion démographique, c'est aussi un moyen d'intégrer les gens à la vie d'une commune, c'est... pas une nouvelle forme, mais ça peut prendre des formes nouvelles qui sont plus attirantes que de s'impliquer dans une asso ou les formes classiques de bénévolat. C'est un nouveau moyen en tout cas. »

H., Enseignant, Bac +3 , plus de 20 ans, Entretien 
Ceux intéressés par un engagement à plus long terme, qui se « renseignent sur les associations communales $\gg^{38}$, présentent leur participation comme un sas d'entrée et suivent une sorte de parcours d'intégration à partir de la cérémonie d'accueil des nouveaux habitants, durant laquelle ils ont découvert ces possibilités de participation qui impulsent une progression par tâtonnement dans la vie locale.

« En tant que nouveaux arrivants, il est encore un peu tôt pour avoir créé de véritables liens. Cela viendra certainement avec le temps.

[...] Nous avons découvert l'existence du forum de démocratie participative lors de la matinée d'accueil des nouveaux arrivants organisée par la commune.

[...] Nous n'avons pas encore participé à une instance de démocratie locale. Notre probable future participation concrétisera notre intérêt pour la politique, cela aura certainement pour effet de renforcer notre intérêt pour la politique.

[...] Un engagement associatif nous intéresserait, car il nous permettrait de connaître du monde ici. »

H., 36 ans, Ingénieur, Bac $+4 / 5$, moins d'un an

Ce profil de l'intégration reste néanmoins minoritaire par rapport au profil de l'enracinement et la prégnance des participants qui s'appuient sur leur ancienneté pour expliquer leur engagement.

\section{Participation de détour et de continuité}

La distinction entre participations de détour et de continuité n'est pas entièrement symétrique de celle entre nouveaux et anciens. Elle renvoie davantage à l'idée de participants de l'extérieur et de l'intérieur. Par cette image, il s'agit ici d'évoquer l'inclusion en cercles concentriques par les dispositifs et les réseaux qui s'entrecroisent dans les trajectoires de participation à la démocratie locale. Si les anciens sont les recrues privilégiées d'une certaine forme de continuité, cela n'exclut pas pour autant des formes faisant davantage référence à l'intégration qu'à l'enracinement. Un tel écart se mesure alors dans le discours entre ceux qui « se retrouvent », ceux qui « s'investissent » dans les dispositifs participatifs et ceux qui «s'y essayent ».

«Cet intérêt existait avant la possibilité de participation à instance de démocratie locale. [...] Je connais les élus et [je voulais] répondre à l'esprit d'ouverture de cette municipalité. »

F., 67 ans, Retraitée (enseignement), Bac, plus de 20 ans

[38] Homme, 25 ans, en recherche d'emploi, Bac +2 . 
" J'étais une citoyenne active ailleurs avant. Je reste attentive et active ici, maintenant. »

F., 46 ans, Pro. intermédiaire, Bac +2 , entre 3 et 5 ans

« J'ai toujours été intéressée par la politique et c'est cela qui m’a donné envie de participer à la démocratie locale [...] J'aimerais être élue dans un conseil municipal. »

F., 40 ans, Cadre, Bac +3 , moins de 3 ans

« J'ai participé par curiosité, pour être au courant de ce qui se dit et fait dans la ville. [...] C'est vrai que j'ai été contente de recevoir une invitation de la mairie pour ce forum. »

F., 83 ans, Retraitée (enseignement), Bac, plus de 20 ans

" [Je voulais] participer et voir au moins une fois à quoi ressemblent les comités participatifs. [Mais] ce n'est pas en allant à une réunion que cela change les idées sur la politique. »

H., 45 ans, Cadre, Bac $+4 / 5$, entre 5 et 10 ans

Cette même étiquette de «participants à » recouvre des réalités différentes qui renvoient à un ordinaire ou à un extraordinaire de l'engagement citoyen. Ces deux termes sont employés pour qualifier le sens que revêt leur participation pour les individus. C'est d'abord la mesure du fait associatif qui est fortement corrélée à un engagement ordinaire. Être membre d'une association est une des caractéristiques les plus partagées parmi les répondants : $71 \%$ affirment leur appartenance à une association, parmi lesquels $56 \%$ se désignent comme « membre actif » ou « organisateur » plutôt que simple « adhérent » dans au moins une association. Cette qualification de leur engagement associatif est d'ailleurs corrélée à leur présence dans les dispositifs participatifs.

Tableau 6. Intensité de la participation selon statut dans au moins une association

\begin{tabular}{|c|c|c|c|c|c|c|}
\hline Statut dans & Nombre moyen & \multicolumn{4}{|c|}{ Participation à « $\mathrm{X}$ » types de dispositifs } & $\%$ de \\
\hline association & citées & 0 & 1 & 2 & $3+$ & $(n=101)$ \\
\hline Non-Bénévole & 0 & $21 \%(6)$ & $32 \%(9)$ & $29 \%(8)$ & $18 \%(5)$ & $28 \%$ \\
\hline Adhérent & 1.88 & $25 \%(4)$ & $25 \%(4)$ & $19 \%(3)$ & $31 \%(5)$ & $16 \%$ \\
\hline Membre actif & 2.00 & $3 \%(1)$ & $17 \%(5)$ & $34 \%(10)$ & $45 \%(13)$ & $29 \%$ \\
\hline Organisateur & 2.04 & 0 & $21 \%(6)$ & $39 \%(11)$ & $39 \%(11)$ & $28 \%$ \\
\hline
\end{tabular}


Les entretiens mettent au jour des positions multiples dans des activités collectives (associations, syndicats, beaucoup plus rarement partis politiques), à l'échelle de la ville, du quartier ou du lotissement. Positions mises en avant pour expliquer leur participation comme part d'un ensemble et qui traduisent une forme de capital social puisqu'elles sont avancées par les acteurs comme un motif d'explication, et non simplement comme une conséquence de leur participation. Les participants interrogés en ce sens décomposent alors les dispositions acquises qui fondent le caractère « ordinaire » de leur participation : expériences associatives, syndicales, interconnaissance par des lieux ou activités communes, enjeu d'accomplissement et de reconnaissance.

«Aller à ce genre de forum, ça fait partie un peu de notre philosophie, de notre façon de voir les choses.

[...] On se connaît un peu, du coup quand il y a quelque chose, on s'adresse à moi...

[...] On avait un stand au forum des associations [...] on rencontre $d u$ monde.

[...] J'ai eu l'occasion de rencontrer le maire à plusieurs reprises, dans d'autres circonstances [...] dans les manifs pour les retraites.

[...] Bouche-à-oreille. [...] Je faisais du covoiturage avec une collègue

[...] qui est élue municipale.

[...] Il y a quand même encore un esprit village. Les gens se connaissent.

[...] On fait des choses [...] très vite on connaît du monde.

[...] Moi, j'aime bien le faire, donc je le fais de bon cœur. Et puis après, je donne des renseignements à qui les veut. »

H., 52 ans, Professeur des écoles, Bac, entre 3 et 5 ans, Entretien

"C'est quelque chose que j'ai acquis moi-même, à partir du syndicat, je pense. Mes parents étaient des cultivateurs, c'était pas... Ils étaient de droite. Le syndicat m’a fait changer, réfléchir à certaines choses. Làj'ai acquis ça. »

F., 74 ans, Retraitée-Fonction publique, CAP-BEP, plus de 20 ans, Entretien

«Ça », à la fois être disposé à et être en position de, c'est ce qui pousse cette membre de Cap à Gauche, déçue de la faible mobilisation pour la démocratie participative, à comparer et juger les différences de fréquentation entre forum citoyen et forum des associations, mais aussi pour le second entre matinée et après-midi.

«Le forum des associations, c'est un lieu pour rencontrer pas mal de gens, je trouve. Il y a plus de monde là. Tout en sachant aussi que beaucoup de gens viennent pour s'inscrire, le matin c'est le rush pour les associations sportives, après on voit plus personne. [...] C'est pas le même public [qu'au forum citoyen], là on retrouve plus 
des gens comme nous, des gens peut-être engagés politiquement. Le forum des associations, c'est un peu dommage on vient chercher un service... »

Et finalement, elle fait une différence entre un engagement associatif et politique local de long terme et une participation au forum citoyen, qu'elle pratique sur le mode de ce que nous appelons ici l'ordinaire : de l'ordre du quotidien du fait des sollicitations et de l'implantation dans les réseaux d'interconnaissance, mais aussi en le valorisant moins que d'autres engagements.

« Le forum en 2012, c'était la première fois. Les autres j'avais pas accroché. [...] J'étais dans [un] groupe [du CCAS], mais le reste non... Le groupe on voit le concret, tandis que quand on va dans le forum, on sait pas trop si ça va aboutir. Et peut-être dans le groupe c'était plus au contact des gens. Je trouvais que le forum... J'avais pas envie d'intégrer en plus encore ça. [En 2012] je me suis décidée parce que j'en ai parlé avec l'adjointe aux affaires sociales, c'est une amie, et aussi peut-être entraînée par [une autre amie] qui est très active. On m’a poussée un peu oui, et les thèmes m'intéressaient plus. »

L'offre de participation est pour une part assimilée à la vie associative, pour ces répondants il y a une équivalence entre milieu associatif et démocratie locale, comme deux facettes d'une même pièce. Interrogés sur les démarches participatives auxquelles ils ont pris part, les participants les plus investis font l'inventaire de l'ensemble de leurs activités locales: le bénévolat au festival de cinéma, la fête des voisins, le pédibus, l'épicerie sociale, la fête de la musique, les journées du patrimoine, le syndic, les jardins partagés...

Dès lors, pour ces participants, les dispositifs participatifs s'intègrent dans un ordinaire de l'engagement. Leur participation aux comités, aux forums, n'est pas vécue comme une parenthèse, et le questionnement visant à caractériser cette participation par rapport à d'autres engagements locaux, notamment associatifs, leur apparaît parfois incongru. Une spécification de la démocratie participative apparaît davantage chez les enquêtés les plus critiques, qui la perçoivent comme une démocratie de proximité procédurière rendant confuse la prise de décision. La non-différenciation s'explique aussi parce que la participation ordinaire s'insère dans des réseaux d'interconnaissance dépassant et précédant les dispositifs. C'est en partie un engagement multi-positionné qui permet d'être au bon moment au bon endroit, et dans les bonnes dispositions, pour être sollicité et accepter de participer. C'est davantage le dispositif participatif qui est venu à eux que l'inverse. Ces participants font ce qu'ils faisaient déjà par ailleurs : retrouver d'autres habitants, participer à des groupes d'échanges, porter des projets... activités désormais regroupées sous le terme de « démocratie locale ».

Cette logique d'extension, de reproduction et de reconversion au sein des trajectoires nuance l'idée d'une distinction définitive entre promoteurs et participants 
des dispositifs participatifs. Il existe ainsi un jeu de va-et-vient de militants reconvertis pour un temps en participants. L'apparente continuité des engagements peut être une image trompeuse et peut renvoyer dans les faits à une succession d'intermittences de la citoyenneté (Carrel, Ion, Neveu, 2009) que le regard rétrospectif et englobant vient lisser dans une continuité. Des participants rapportent ainsi les échecs antérieurs qu'ils ont pu connaître : une association de quartier, un système d'échange local, qui se sont essoufflés. L'offre de participation municipale leur pourvoit alors un cadre pour renouer avec l'engagement, apartisan et confortable, en les soulageant de la responsabilité de la mobilisation, de l'animation et de la fidélisation avec les bénévoles.

«Cette participation ne fait qu'illustrer mon intérêt pour la politique, mais elle aide aussi à aller de l'avant, car il n'est pas toujours évident de s'engager. »

F., 67 ans, Retraitée, Bac $+4 / 5$, plus de 20 ans

Le questionnement autour d'une demande sociale de participation révélée par les dispositifs participatifs ne résonne alors réellement que lorsqu'il rencontre des participants dans une trajectoire ascendante d'engagement dans la vie locale, tendanciellement plus jeunes et plus récemment arrivés dans la commune, chez qui l'idée de facilitation de l'engagement est également repérable. Ainsi, une trentenaire, arrivée depuis trois ans dans la ville, évoque une première tentative abandonnée dans un réseau militant écologiste, qu'elle compare avec l'engagement dans les comités consultatifs sur le développement durable que depuis elle privilégie. Par la suite, elle rejoint d'autres groupes sur les déplacements, participe au forum citoyen et se voit proposer de participer aux ateliers de réflexion pour l'élaboration du programme et d'être sur la liste municipale.

« Je me sens mieux dans ce groupe-là [plutôt que dans] un groupe qui était pas en plus très constitué, qui marchait pas depuis longtemps, donc qui était en train de trouver ses marques, alors que les ateliers je m'y suis sentie à l'aise, parce que c'est structuré. Les deux élus font avancer la réunion de façon constructive, on n'est pas juste là à parler dans le vent. [...] Il y [a un] cadrage agréable qui fait que c'est pas seulement celui qui a plus de bagout qui va prendre toute la parole. »

\section{F., 29 ans, médical, Bac $+4 / 5$, moins de 3 ans, Entretien}

La poursuite de l'engagement dans ces trajectoires cumulatives tient à la rencontre entre des conditions facilitantes offertes par l'offre de participation et une envie d'engagement préalable, motivée à la fois par un désir d'intégration et un intérêt pour un thème. Cette description d'un échange entre offre et demande de participation suppose ainsi des dispositions et des disponibilités qui s'actualisent à travers l'opportunité de participation. Ce schéma est d'autant plus visible lorsqu'en contraste sont interrogées des personnes pour qui la présence à une instance de démocratie participative a constitué un détour très circonstancié, 
une parenthèse depuis refermée. Des habitants qui n'ont pas par ailleurs d'engagements similaires, notamment associatifs, et qui sont donc moins intégrés dans ces réseaux de sociabilité, vecteur de sollicitations incitant à la réitération des participations extraordinaires.

" Je ne suis pas une grande bavarde. [...] J'ai pas grand-chose à dire, moi si je l'ai fait c'était surtout la réunion au niveau des jeux, pour les enfants il n'y avait que ça qui m'intéressait dans l'immédiat, après le reste m'intéressait pas, tout ce qu'ils proposaient à la mairie, il n'y avait juste que cela. Le but des jeux, pour les petits que je garde [en tant qu'assistante maternelle]. [...] Je me suis lancée là-dedans, autrement moi je suis pas quelqu'un qui va dans des réunions comme ça. Le sujet me parlait, c'est pour ça que j'y suis allée, autrement non, je suis pas du style à aller là. [...] Le forum, j'y suis pas allée, le tour de table, comme ça, par petits groupes, et puis les sujets abordés, non, je ne me voyais pas, je ne voyais pas ce que je pouvais leur apporter. J'ai laissé la place aux autres. »

F., 50 ans, Assistante maternelle, CAP-BEP, moins de 3 ans, Entretien

[Je suis allée au forum] car j'étais directement concernée par ce sujet : nouveau quartier, nouveaux résidents, mauvaise acceptation. [Je voulais] rencontrer les gens concernés eux aussi par ce sujet. [...] Honnêtement je n'ai pas trouvé une grande implication de la part des habitants. Les élus n'ont pas su me motiver pour revenir cette année. [Je ne suis] pas certaine que les différents points de vue soient pris en compte. [...] 2012 a été un tournant puisque mes idées ont évolué. C'est la première fois que je votais à droite.

F., 45 ans, Secrétaire, Bac, entre 3 et 5 ans

De manière imagée, ces catégories de l'ordinaire et l'extraordinaire (cf. tableau 7) décriraient des gymnastes, certains rompus à l'exercice, d'autres débutants, mais tentant chacun à leur niveau d'effectuer la même action : sauter. Les uns le faisant sur une série de trampolines sur lesquels ils rebondissent directement passant de l'un à l'autre (2.1) et les autres le faisant grâce à ce nouveau tremplin sur lequel ils devront d'abord remonter après avoir repris leur élan pour ressauter. Une partie s'en détournera (1.1) quand une autre tentera de persévérer jusqu'à s'essayer aux trampolines (1.2). Enfin, ce tremplin mis à disposition par l'offre de participation, certains militants n'en ont guère l'usage (2.2) et restent sceptiques quant à son intérêt, sautant déjà suffisamment haut ou loin. Dans leurs cas, ce sont aussi leurs propres capacités à pouvoir le contourner, voire à le détourner, par la pétition ou la demande de constitution d'espaces participatifs ad hoc, qui fondent les « ressorts » d'une adhésion distanciée (Rui, VillechaiseDupont, 2006). 
«Le fait que je suis déjà engagé auprès d'associations de la ville fait que je me sens concerné par les décisions de la municipalité. » H., 59 ans, Employé, Brevet, natif

« J'ai besoin de digérer une déception latelier parentalitél avant de me réinvestir dans une autre action (comité d'usagers de la médiathèque). »

F., 56 ans, Retraité, Bac +3 , entre 10 et 20 ans

Les différents dispositifs proposés à la population dessinent les étapes d'une carrière citoyenne qui implique potentiellement une progression dans l'intensité de l'engagement, et pour certains une montée en généralité depuis les problèmes locaux, les enjeux municipaux, jusqu'à une mise en perspective des limites des compétences municipales dans le contexte de l'agglomération. Cette image est aussi un effet de l'offre : un comité consultatif étant par la suite transformé en comité d'usagers, suite à la réalisation d'un équipement ou après la formulation d'un diagnostic. Mais pour les individus, l'image de carrière peut être trompeuse, car elle sous-entend l'idée d'une progression. Une telle dynamique est minoritaire, souvent le détour participatif se renouvelle au même niveau d'engagement, par la fréquentation d'un groupe devenu familier, ou ne se renouvelle pas. De là vient cette image ironique d'une participante au forum désignant le «fan-club des élus », au sens où il y a un noyau caractérisé par une forte interconnaissance et un engagement qui ne se limite pas aux dispositifs participatifs, pour qui les instances de démocratie locale sont une composante d'une participation plus large à la vie municipale.

Des dynamiques de politisation opèrent à la marge, parmi ceux qui font plus directement le lien entre leur participation et la perspective d'une action politique locale, de laquelle ils se sentent potentiellement parties prenantes au fil du temps. Ainsi, certains, par ces détours, lorsqu'ils viennent compléter ou supplanter un autre engagement, s'investissent dans une logique de carrière citoyenne (Birck, 2011).

" Je ne suis pas engagée, mais l'idée mûrit [...] participer plus à des réunions publiques, me rapprocher des élus »

F., 47 ans, comptable, Bac +2 , entre 10 et 20 ans

«On peut donner son avis en sachant qu'il va être pris en compte dans les discussions. On se sent utile pour la ville. [...] J'ai plus tard envie de prendre des décisions et de m'investir politiquement [...] je suis plus motivée encore pour participer. »

F., 18 ans, étudiante DUT, native 


\begin{tabular}{|c|c|c|c|c|c|c|c|}
\hline 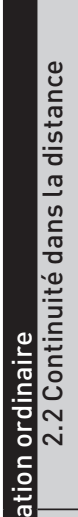 & 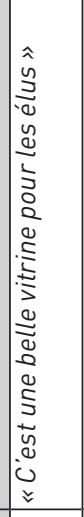 & 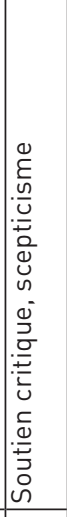 & 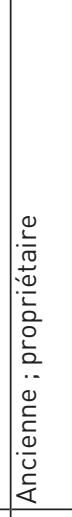 & 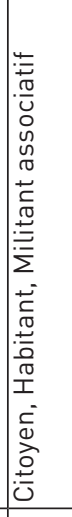 & 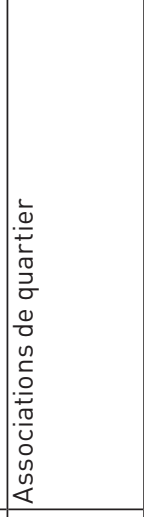 & 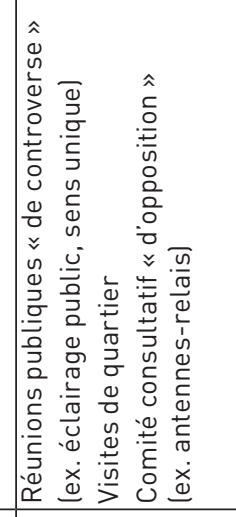 & 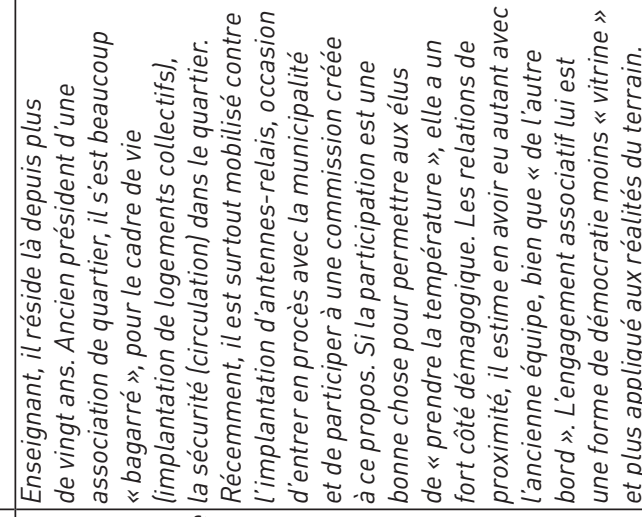 \\
\hline 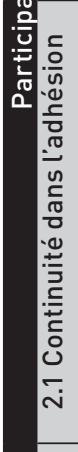 & 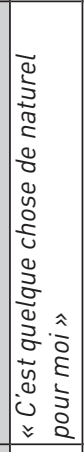 & 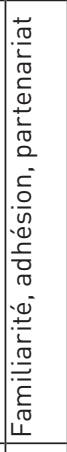 & 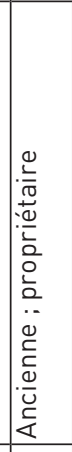 & 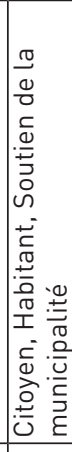 & 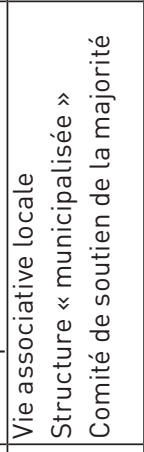 & 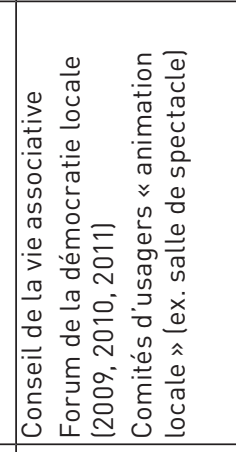 & 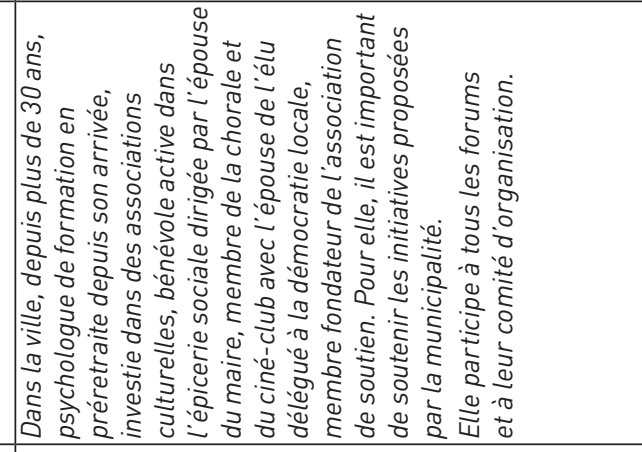 \\
\hline 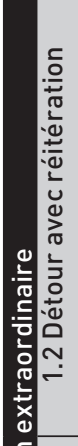 & 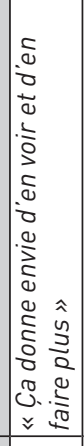 & 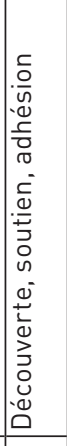 & 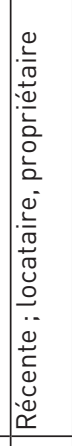 & 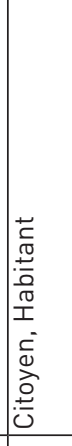 & 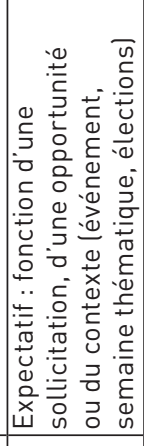 & 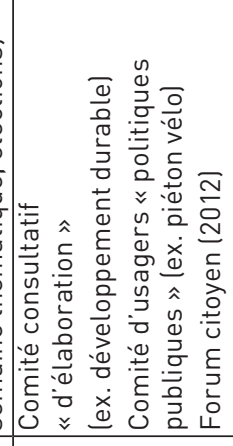 & 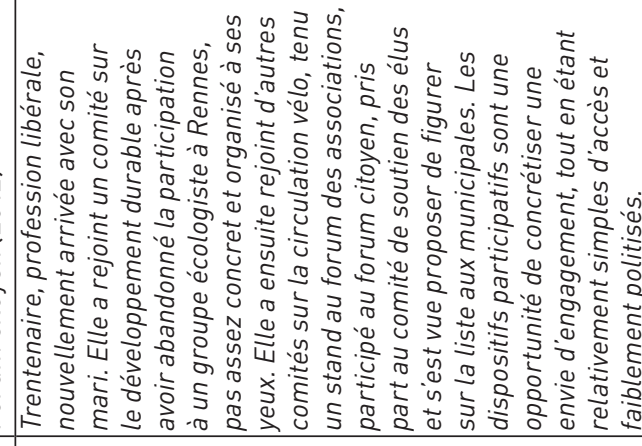 \\
\hline 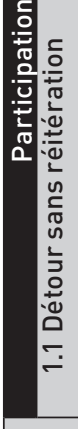 & 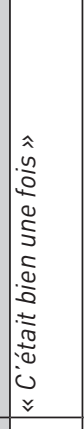 & 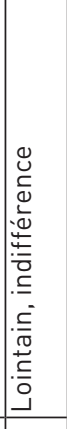 & 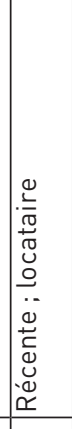 & 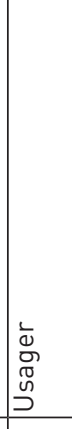 & & 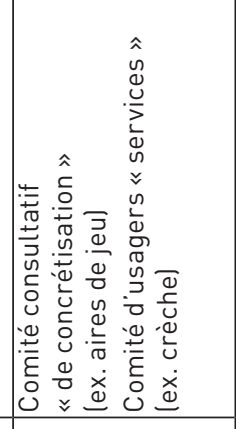 & 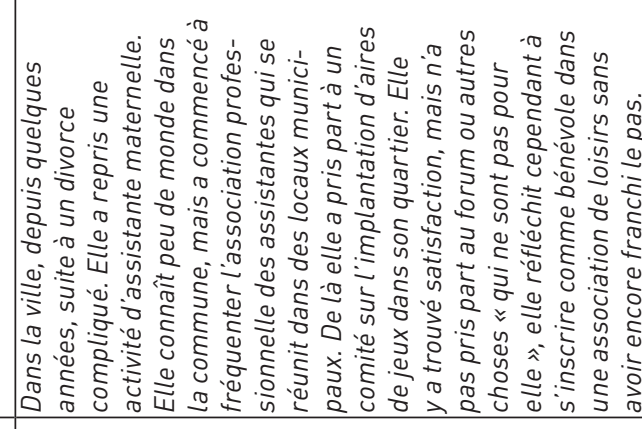 \\
\hline 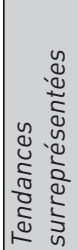 & 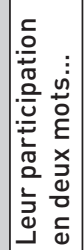 & 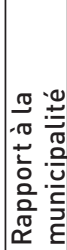 & 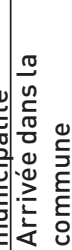 & |ֶ, & 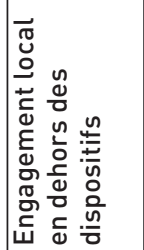 & 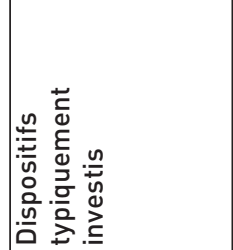 & 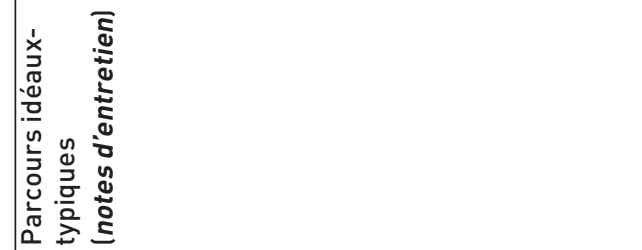 \\
\hline
\end{tabular}


" Ça donne envie de la politique finalement [...] pour moi c'était réservé à une élite bourgeoise et à des beaux-parleurs. Mais la démocratie participative, ça donne l'espoir que tout le monde peut participer. [Pourquoi pas] un engagement dans la prochaine équipe municipale? Mais pas dans un parti politique national, car là je n'ai pas trop d'espoir qu'on puisse faire quelque chose. »

F., 29 ans, médical, Bac $+4 / 5$, moins de 3 ans

Ces participants extraordinaires gèrent leur entrée en participation et son éventuelle reconduction par la mobilisation de différentes ressources, quand par exemple une démarche d'accession à la propriété, une habitude de travail selon des modalités proches ou un intérêt acquis pour des thématiques comme le développement durable ou les politiques culturelles, les incitent à approfondir leur engagement.

«Par rapport au développement durable, moi je suis assez sensibilisée à ça parce que j'ai mis en place les certifications ISO 9001 et 14001 au sein [de mon cabinet], et du coup je voulais voir si d'autres entreprises étaient dans cette démarche sur la commune. C'est pour ça que j'ai intégré le comité. [...] J'ai aimé participer à une action pour la ville, c'était une des premières fois que je m'investissais au niveau de la mairie, pour la ville. »

F., 53 ans, chargée de communication, Future membre du comité de soutien, Entretien

Ces dynamiques de politisation par la participation, ou du moins de liens entre expérience de la participation et rapport au champ politique local contrastent avec un rapport de défiance s'exprimant en dehors de toute participation aux dispositifs ou aux associations.

" Je crois que je ne voterai plus, car la démocratie n'existe pas. [...] Je suis un ouvrier alors je pensais quavec la gauche, il y avait un espoir de mieux vivre, mais ce n'est pas le cas. [...] j'habite près d'un centre commercial qui provoque quelques nuisances de bruits [...] Je suis allé voir le maire. Il constate qu'il y a gêne, mais ne peut rien faire. [...] Solidarité ? Quel beau mot : même mes voisins ont refusé de signer ma pétition!»

$H$., 59 ans, ouvrier au chômage, CAP-BEP, entre 10 et 20 ans

Deux logiques semblent ainsi ressortir. D'une part, les habitants qui s'appuient sur leur enracinement : des retraités, investis dans plusieurs associations, revendiquant une culture de l'engagement et qui se retrouvent dans les dispositifs participatifs, tendanciellement ceux s'inscrivant dans une démocratie de proximité (visites de quartier, réunions publiques, commissions municipales). Et d'autre part, des actifs, aux niveaux de diplôme et de revenu plus élevés, et qui investissent d'abord des espaces comme les premières éditions des forums et 
des dispositifs fonctionnant davantage sur le mode du groupe de travail : comités d'usagers, comités consultatifs, ateliers thématiques. C'est alors par des voies différentes qui se superposent dans les dispositifs participatifs, que leur présence en tant que participant «va de soi », qu'il s'agisse de s'y retrouver ou de s'y investir. C'est d'ailleurs le cumul de ces formes de ressources et de légitimités qui caractérisent les participants les plus investis et reconnus.

« Je suis natif de la commune, mes parents [y] étaient commerçants. [...] Je connais vraiment la ville, et j'ai la chance de rénover une longère dans mon quartier [...] En septembre on y fait un festival [...] je suis trésorier de l'association [...] J'ai toujours été investi dans l'associatif. J'ai fait ma scolarité dans cette école [...] j'ai enseigné trente ans à Rennes et maintenant j'y suis revenu. [...] Je suis investi dans le foot depuis 45 ans dans la commune, donc je connais forcément du monde. Je suis responsable de la comm' avec Ouest-France [...] Et là je soutiens la liste et je suis dans l'associatif de quartier. [...] J'ai participé au forum en tant qu'animateur les deux premières années. [...] J'ai toujours eu une sensibilité de gauche, je me suis présenté aux élections municipales il y a 25 ans déjà. Après j'ai toujours soutenu. » H., 51 ans, Professeur des écoles, Bac, Futur président du comité de soutien, Entretien

\section{De quoi la demande sociale est-elle le nom ?}

«Si on fait pas attention, il n'y a pas une demande de participation. Par contre, il y a une demande de résoudre les questions avec leur avis. Ce qui revient à peu près au même si on décode... Mais c'est à ça qu'il faut qu'on soit attentif. [...] souvent ça mamène à retourner la question, à décaler la prise de décision... de façon à faire rentrer les gens dans une démarche plus collective [...] C'est une façon de gérer la diversité des avis ou les contradictions d'avis. Parce que ce qu'on nous reproche très souvent, globalement dans ces démarcheslà... c'est "oui, vous vous appuyez sur quelques personnes, que vous connaissez...", alors, ça on peut montrer que non. [...] Et si nous on a ce souci d'aller chercher les autres et d'aller causer aux autres, je pense que, pour une part, on rentre dans ce cycle, que j'estime vertueux, de la construction collective. Dont la participation est un des modes parmi d'autres. »

Entretien, maire

La pertinence de la catégorie de « demande sociale » est en partie remise en cause par l'analyse des motifs d'engagement et des parcours dans la démocratie locale. Ce qui s'observe, ce sont des dispositions acquises confrontées à des opportunités qui constituent des contextes d'actualisation (Mathieu, 2012). Des dispositions qui majoritairement sont patentes chez les participants qui vivent 
leur participation sur le mode de l'ordinaire, et qui ne sont que minoritairement latentes chez ceux pour qui elles constituent un détour qui n'est réitéré que marginalement.

Au fil de l'enquête, des groupes d'individus d'abord considérés comme des potentiels informateurs pour interroger la demande semblent se situer davantage du côté de l'offre. Lier la qualité de demandeur à la position de participants, c'est jeter un voile sur le fait que ces acteurs sont souvent proches des offreurs à cette échelle communale, en termes de profils sociologiques, de réseaux et de trajectoires. Cette proximité s'incarne dans une interconnaissance de longue date et/ou des occupations bénévoles fortement intégrées ou assimilées à la municipalité (CCAS, office du tourisme, épicerie sociale...), dans une participation au comité de soutien électoral et à la marge dans une figuration sur la liste électorale $^{39}$. Pour le regard extérieur, il semble s'en être fallu ou s'en falloir de peu pour que ces engagés « ordinaires » soient plus que des participants, si ce n'est, au-delà des contingences, la propre compréhension qu'ils donnent à leur engagement à travers leurs redéfinitions du politique et les normes de citoyenneté qu'ils mobilisent, faisant notamment d'un engagement partisan une ligne jaune.

Les opportunités de participations revalorisent certains types d'échanges et d'engagements qui leur préexistent. En pensant cet échange à travers l'hypothèse d'une demande de participation, l'analyse se concentre sur l'étude de variations dans des trajectoires extraordinaires, qui sous-entendent une demande latente qui serait révélée, mais elle délaisse l'ordinaire qui concerne pourtant la majorité des participants, ou alors elle tend à juger cette disproportion comme un échec d'une participation peu inclusive. L'offre de participation locale, ou plus exactement l'extension et l'évolution des opportunités de participation à la vie locale sous différentes formes, tant via des associations que des dispositifs participatifs dans le contexte d'une alternance politique, fonctionne ainsi davantage comme un système de vases communicants, où l'offre est un cadre dans lequel une majorité d'engagements préalables et une minorité d'engagements nouveaux peuvent s'épanouir.

Les participations extraordinaires émergent en réponse à un certain cadrage de l'offre, comme l'illustre le repositionnement du dernier forum ou des comités mobilisant davantage la figure de l'usager que du citoyen ou se concentrant sur des thématiques perçues comme immédiates et concrètes. Deux pôles en déséquilibre se distinguent ainsi, entre un détour participatif et une participation

[39] Figuration au sens où il semble s'agir plus d'un soutien affiché que d'une envie d'élection. En effet, parmi les candidats en 2014, une douzaine ne fait pas partie de l'équipe élue en 2008 : la moitié apparaît dans la population construite pour notre enquête par questionnaire. Cet indice est d'ailleurs corrélé au positionnement des nouveaux candidats en 2014 sur la liste : la place moyenne sur la liste des six «participants » (i.e. référencés dans la population pour l'envoi de notre questionnaire) est de $27 / 33$ (max. 20 ; min. 33), celle de ceux n'y figurant pas est de 22/33 (max. 12 ; min. 31), le premier sous-groupe étant davantage homogène que le second. 
s'inscrivant dans une continuité. Le premier peut s'arrimer à des motivations liées à l'individu ou à son premier cercle de sociabilité, notamment familial ou professionnel, quand la seconde mobilise davantage les références à un collectif ou une communauté, signe d'une sociabilité acquise notamment dans l'engagement associatif local. Ces appropriations différenciées d'une offre de participation traduisent aussi « au nom de quoi » elles se font (Hatzfeld, 2011). Ceux que l'offre de participation mobilise le plus efficacement, sont des offreurs intermédiaires de participation, qui s'inscrivent dans cette offre comme des relais et mettent en avant une idée active de la citoyenneté dont ils retirent des gratifications symboliques. L'offre de participation est perçue comme un bien venant en articulation avec un capital déjà existant : consommer ou s'équiper de ce bien ne fait sens à long terme qu'en disposant de ce capital ou en l'acquérant.

Les participants ordinaires prennent au sérieux leur métier de citoyen et mettent en avant leurs engagements antérieurs, qui les amènent à être présents dans les dispositifs, tout en relativisant la portée des engagements dans les dispositifs participatifs qui restent secondaires dans leurs trajectoires. Les participants extraordinaires sont ceux qui ont peu d'engagements durables par ailleurs, du fait d'une arrivée récente, d'une moindre disponibilité ou d'un sentiment d'incompétence. Ils s'engagent pour une participation précise, délimitée, et peuvent en attendre un gain potentiel, mêlant intérêt, connaissance et reconnaissance, et a contrario sont hésitants à aller au-delà et potentiellement déçus. Les conditions de l'accès à ce détour participatif sont liées à des trajectoires lexpériences associatives, syndicales, travail en lien avec le sujet...) et à des moments propices au sein de celles-ci (nouvelle ville, nouveau métier, temps libre). Au-delà, les trajectoires de participation qui s'étirent et se renouvellent doivent pouvoir se satisfaire d'autres motivations liées à une vision engagée de la citoyenneté, et qui peuvent s'interpréter en dernière instance comme une « demande de participation ».

La démocratie participative n'en permet pas moins une diversification des parties prenantes dans les discussions sur l'espace public local. Par ailleurs, certains dispositifs, relevant de la «petite démocratie » (Mothé, 2006), réussissent à s'ancrer dans un quotidien et toucher en partie un autre public. Ce partage ponctuel est d'ailleurs assumé par le maire pour qui au moins : « [ces] gens ont peut-être décidé quelque chose dans leur vie et ont l'impression d'avoir partagé ». Ici, l'important est peut-être davantage d'avoir pu participer à court terme et directement à une décision, d'avoir pu s'insérer dans un groupe où ils ont le sentiment «[d'avoir] moins de chances de [se] tromper, que dans des choses où on peut parler des impôts, genre de conversations [où ils ne s'] aventureraient pas $\gg^{40}$.

[40] Entretien, habitante. 


\section{Conclusion}

Si le volontarisme politique de l'équipe municipale a pu amplifier la capacité d'écoute de l'institution, cette volonté reste largement tributaire de l'inégale répartition dans l'espace social des dispositions que l'offre de participation se propose d'actualiser. La mise en place d'une offre participative institutionnelle à l'échelle d'une ville s'inscrit dans un contexte, et l'enquête met ainsi au jour la rencontre entre un état préexistant de la vie associative, une interconnaissance relativement élevée et de nouveaux enjeux liés à l'agrandissement de la ville, l'intégration de populations, qui viennent en partie donner du sens à une offre de participation. Pourtant, le choix de promouvoir différentes possibilités de participation à travers une gamme d'opportunités fait partiellement sens, les profils extraordinaires décrits étant en accord avec cette intention première. Il y a en partie par ces modes de gestion municipaux une concrétisation d'« usages inclusifs de la représentation » (Hayat, 2013).

Cette proposition institutionnelle ne s'affranchit pas pour autant de limites connues par ailleurs : légitimité du représentatif dans l'espace public (Gaxie, 2004), absorption de la participation dans la représentation (Blatrix, 2009), faiblesse démocratique de la décentralisation (Paoletti, 2007), inégale répartition des dispositions à investir les questions politiques (Gaxie, 1978 ; Bourdieu, 2000). Ces enjeux structurels rappellent que « la démocratie participative n'a de sens que si elle contribue à enrayer les logiques d'exclusion sociale qui caractérisent aujourd'hui le fonctionnement ordinaire de nos démocraties » (Blondiaux, 2008a, p. 109), mais que si une offre de participation descendante se confronte à ces logiques, les intègre et entend en partie y répondre, elle ne les dépasse que partiellement, sous conditions, en interaction avec un contexte et des dispositions. L'aspect « ordinaire » se retrouve pour une part dans une participation qui répond à ce même qualificatif, et qui recouvre la majorité des engagements qui s'arriment aux dispositifs participatifs et leur permettent d'exister. Ce constat de continuité et de reversement des engagements ne doit pas pour autant occulter les possibilités d'une participation qui sort de l'ordinaire, mais il en rappelle néanmoins le caractère forcément conditionné et fortement circonscrit. Notre étude révèle des appropriations différenciées d'une offre de participation institutionnelle, depuis une participation dans la continuité d'un engagement plus large, qui s'ajoute à une liste ouverte, jusqu'à un détour ponctuel, qui s'insère dans une parenthèse pour un temps refermée.

Au-delà du périmètre de notre recherche, la coexistence de différentes logiques d'engagement parmi les publics participants est le reflet d'un débat entre deux conceptions des politiques de participation publique : un néocorporatisme assumé avec les corps intermédiaires ou une démocratie participative inclusive avec les citoyens ordinaires ${ }^{41}$. Mais encore faut-il ici distinguer l'analyse de ce qu'elles

[41] Nous reprenons ici les termes du «face à face » publié sous forme de tribunes croisées de G. Gourgues et J. Talpin dans la revue du centre de ressources prospectives du Grand Lyon : M3 Société urbaine et action publique, n 7, 2014, p. 30-33. 
sont et la réflexion sur ce qu'elles pourraient ou devraient être. L'observation des appropriations différenciées d'une offre de participation fait ainsi percevoir les difficultés d'équilibrage entre un public à satisfaire et un public à mobiliser. Les militants associatifs se sont adaptés à une offre de participation que certains ont eux-mêmes contribué à faire émerger, dans le contexte d'une alternance municipale dont ils étaient pour une grande part les soutiens. Ceux-là y voient la possibilité concrétisée d'un rôle de partenaires qui leur était auparavant refusé, tandis que ceux qui restent plus critiques y décèlent davantage un filtre supplémentaire dans l'accès aux élus. Les autres, les citoyens profanes à la participation extraordinaire sont ceux qui s'y sont retrouvés après coup et chez qui la réitération suppose l'acquisition d'un capital social par l'actualisation de dispositions préalables.

Dans le contexte d'une difficile mobilisation des publics, illustrant une absence de demande sociale de participation, en tout cas l'absence de conditions rendant sa possibilité effective, l'expérience de la différenciation des modes de participation semble être une réponse partielle. Au-delà des logiques observables d'homo- et d'endogamie, le cas des participants extraordinaires illustre finalement le dilemme et la contradiction des politiques de participation publique, puisque leur implication est posée comme un critère de réussite des dispositifs participatifs, quand dans le même temps leur rapport à ces dispositifs, par leur absence remarquée ou leur présence remarquable li.e. qui possède des propriétés que tous les éléments analogues ne possèdent pas), en est un révélateur des limites intrinsèques. Pourquoi dès lors s'intéresser aux offres institutionnelles de participation? D'abord parce qu'elles ne constituent pas, ni pour leurs promoteurs, ni pour leurs publics, une réalité univoque. Les appropriations dites différenciées sont en soi des indices permettant de comprendre les motifs et les conditions sociales des engagements participatifs, et à travers eux les contours d'une demande de participation, et comment l'offre la façonne et s'en trouve façonnée. Que les participations relèvent d'un ordinaire tracé ou d'un extraordinaire déroutant, elles s'insèrent dans des trajectoires contextualisées qui demandent dans des développements ultérieurs leur prise en compte longitudinale, mais aussi la comparaison avec d'autres cas où l'offre de participation prend une forme différente. 


\section{Bibliographie}

Anquetin V., Freyermuth A., 2008, La figure de l'« habitant». Sociologie politique de la demande sociale. Rennes, Presses universitaires de Rennes.

Bengtsson A., Christensen H. S., 2013, « Do citizens' patterns of political participation correspond to their process preferences », EPOP 2013, 13-15 September, Lancaster University.

Birck N., 2011, « Le défaut d'inclusion interne des dispositifs participatifs. Vers l'émergence d'une nouvelle élite citoyenne ? », communication à la seconde journée doctorale sur la participation du public et la démocratie participative, Paris, EHESS, 18 octobre 2011.

Blatrix C., 2000, La démocratie participative, de mai 68 aux mobilisations anti-TGV. Processus de consolidation d'institutions sociales émergentes, thèse pour le doctorat de science politique, Université Paris I - Panthéon Sorbonne.

Blatrix C., 2001, « Actions associatives et transformations des démocraties : en prenant les détours participatifs », colloque à l'université de Saint-Étienne, Actions associatives - solidarités et territoires, 18-19 octobre 2001.

Blatrix C., 2009, « La démocratie participative en représentation », Sociétés contemporaines, 74, p. 97-119.

Blondiaux L., 2004, « L’idée de démocratie participative : enjeux, impensés et questions récurrentes », conférence à l'Université du Québec, Montréal, 11 novembre 2004.

Blondiaux L., 2007, «Faut-il se débarrasser de la notion de compétence politique ? Retour critique sur un concept classique de la science politique », Revue française de science politique, 57 (6), p. 759-774.

Blondiaux L., 2008a, Le nouvel esprit de la démocratie, Paris, Seuil.

Blondiaux L., 2008b, « Démocratie délibérative vs. démocratie agonistique ? Le statut du conflit dans les théories et les pratiques de participation contemporaines », Raisons politiques, 30, p. 131-147.

Bolzendahl C., Coffe H., 2013, « Are 'Good' Citizens 'Good' Participants? Testing Citizenship Norms and Political Participation across 25 Nations », Political Studies, 61 (S1), p. 45-65.

Bourdieu P., 2000, Propos sur le champ politique, Lyon, Presses universitaires de Lyon.

Bowler S., Donovan J., Karp K., 2007, « Enraged or Engaged? Preferences for Direct Citizen Participation in Affluent Democracies », Political Research Quarterly, 60 (3), p. 351-362.

Carrel M., 2009, « La citoyenneté plurielle. Appréhender les dispositifs participatifs dans leur environnement », in M. Carrel, C. Neveu et J. Ion (dir.), Les intermittences de la démocratie. Paris, L'Harmattan, p. 89-100.

Carrel M., 2013, Faire participer les habitants. Citoyenneté et pouvoir d'agir dans les quartiers populaires, Paris, ENS Éditions.

Crozier M., Friedberg E., 1977, L'acteur et le système, Paris, Seuil. 
Dalton R., 2007, The Good Citizen: How Young People are Transforming American Politics, Washington DC, CQ Press.

Dalton R., 2008, « Citizenship Norms and the Expansion of Political Participation », Political Studies, 56 (1), p. 76-98.

Gaxie D., 1978, Le cens caché, Paris, Seuil.

Gaxie D., 2004, « La démocratie participative dans les structures de la démocratie représentative », in D. Damamme (dir.), La démocratie en Europe, Paris, L'Harmattan, p. 41-50.

Gourgues G., Sainty J., 2011, « La démocratie participative peut-elle convaincre la population de participer? Analyse d'une enquête par sondage », communication à la journée d'études sur les effets de la participation, Paris, EHESS, 21 octobre 2011.

Gourgues G., 2012, «Avant-propos : penser la participation publique comme une politique de l'offre, une hypothèse heuristique », Quaderni, 79, p. 5-12.

Gourgues G., Rui S., Topçu S., 2013, « Gouvernementalité et participation », Participations, 6, p. 5-33.

Gourgues G., 2014 «Pour un néocorporatisme assumé », M3 - Société urbaine et action publique, 7, p. 30-32.

Hatzfeld H., 2011, Les légitimités ordinaires. Au nom de quoi devrions-nous nous taire? Paris, L'Harmattan, Adels.

Hayat S., 2013, « La représentation inclusive », Raisons politiques, 50, p. 115-135.

Hibbing J., Theiss-Morse E., 2002, Stealth Democracy, Cambridge, University Press.

Hibbing J., Theiss-Morse E., 2005, « Citizenship and Civic Engagement », Annual Review of Political Science, 8, p. 227-249.

Jallon H., Manin B., 2001, « La démocratie n'est pas un système apaisé », Mouvements, 18, p. 13-20.

Lefebvre R., 2004, «Quand légitimité rime avec proximité », Mouvements, 32, p. $135-138$.

Lefebvre R., 2007, «Entre démocratie d'opinion et démocratie participative », Nouvelles Fondations, 7-8, p. 20-29.

Lefebvre R., 2012, « La démocratie participative absorbée par le système politique local », Métropolitiques, http://www.metropolitiques.eu/La-democratieparticipative.html, 29 octobre 2012.

Mathieu L., 2012, L'espace des mouvements sociaux, Paris, Éditions du Croquant.

Mazeaud A., 2010, La fabrique de l'alternance. La démocratie participative dans la recomposition du territoire régional (Poitou-Charentes 2004-2010), thèse pour le doctorat de science politique, Université de La Rochelle.

Mazeaud A., Talpin J., 2010, «Participer pour quoi faire ? Esquisse d'une sociologie de l'engagement dans les budgets participatifs », Sociologie, 1 (3), p. 357-374. 
Mothé D., 2006, « La grande démocratie et la petite démocratie », Esprit, 7, p. 35-53.

Neblo M., Esterling A., Kennedy R., Lazer D., Sokhey A., 2010, « Who Wants to Deliberate - And Why ? », American Political Science Review, 104 (3), p. 566-582.

Paoletti M., 1997, La démocratie locale et le référendum, Paris, L'Harmattan.

Paoletti M., 2007, Décentraliser d'accord, démocratiser d'abord, Paris, La Découverte.

Premat C., 2009, « The implementation of participatory democracy in French communes », French politics, 7, p. 1-18.

Putnam R., 2000, Bowling Alone - The Collapse and Revival of American Community, New York, Simon \& Schuster.

Renahy N., «Classes populaires et capital d'autochtonie. Genèse et usages d'une notion », Regards sociologiques, 40, 2010, p. 9-26.

Retiere J.-N., 2003, « Autour de l'autochtonie. Réflexions sur la notion de capital social populaire. », Politix, 16 (63), p. 121-143.

Richard-Ferroudji A., 2011, « Limites du modèle délibératif : composer avec différents formats de participation », Politix, 96 (4), p. 161-181.

Rosanvallon P., 2006, La contre-démocratie, Paris, Seuil.

Rui S., Villechaise-Dupont A., 2006, « Les associations face à la participation institutionnalisée : les ressorts d'une adhésion distanciée », Espaces et sociétés, 123, p. 21-36.

Talpin J., 2011a, Schools of democracy, Colchester, ECPR Press.

Talpin J., 2011b, « Retour sur la politisation des individus par la participation. Pour une approche pragmatique des effets de l'engagement participatif sur les acteurs », communication à la journée d'études sur les effets de la participation, Paris, EHESS, 21 octobre 2011.

Talpin J., 2014, « Pour une démocratie participative inclusive », M3 - Société urbaine et action publique, 7, p. 31-33.

Talpin J., Sintomer Y., 2011, «Équiper ou dépasser la proximité ? Les pratiques participatives régionales à la lumière de l'expérience de Poitou-Charentes », in J. Talpin, Y. Sintomer (dir.), La démocratie participative au-delà de la proximité. Le Poitou-Charentes et l'échelle régionale, Rennes, Presses universitaires de Rennes, p. 11-26.

Webb P., 2012, « Who is willing to participate, and how? Dissatisfied democrats, stealth democrats and populists in the UK », Sussex European Institute, Working Paper No. 131. 


\section{Abstract - Patterns of participation in local democracy: in the ordinary and out of the ordinary course of commitment}

What does a municipal offer of participation mean and imply? This paper questions how such offer can be convincing - under what conditions and to what point - even though participants embrace institutional offer differently. The analysis of the multiple individuals' trajectories and positioning among the public of participatory devices helps to understand how what is said to be a political innovation is also anchored in an ordinary state of local involvement. From that point this paper stresses the stated conditions for a participatory movement for a participatory movement that are not only prefigured in a preexisting environment. That is what is said as patterns of participation in the ordinary and out of the ordinary course of commitment. This research in progress is part of a larger concern about hypothetical social claims for participation, and how such claims are constructed and taken in account by participatory devices. A better understanding of how a granted participatory opportunity is or is not grasped by some citizens allows to gain access to a contextual range of answers about people's preferences regarding political process and how they want to be involved in it.

Keywords participatory democracy, public participation, local politics, publics, social demand, motives

Guillaume Petit est doctorant en science politique (CESSP-CRPS, Université Paris 1, Panthéon-Sorbonne), il réalise sa thèse dans le cadre d'une convention Cifre au sein d'un cabinet de conseil. Ses recherches portent sur les logiques et les enjeux politiques et sociaux d'offres de participation publique locales et sur les caractéristiques et les trajectoires des participants. Il interroge dans ses travaux la notion de demande sociale de participation, au regard des motifs de l'engagement participatif.

\section{Mots clés}

démocratie participative, participation publique, politique locale, publics, demande sociale, motifs 